\title{
THE PROPAGATION OF WAVE-MOTION IN AN ISOTROPIC ELASTIC SOLID MEDIUM
}

\author{
By A. E. H. Love. \\ [Receired nnd Read November 12th, 1003.] \\ CONTENTS.
}

1. Introductory.-2. Notation.-3. Conditions to be satisfied at a Wave-Boundary.4. Displacement due to Body Forces. - 5. Potentials of Disturbance due to Force at a Point.6. Effect of Force at a Point.-7, 8. Establishment of the State of Strain due to Constant Force at a Point.-9. Graphic Representation of the Establishment of the Strain due to Constant Force at a Point.-10. Momentary Force.-11. Double Forces.-12. Generalized Double Forces.13. Multiple Forces.-14-17. Extension of Kirchhoff's Theorem.-18. Disturbance due to an Initial Stato.-19. Potentials of Disturbance due to Initial Velocity.-20. Rules of Differentiation.-21. Differentiation of Surface Integrals.-22. Displacement due to Initial Velocity.23. Poisson's Integral Formula.-24, 25. Displacement due to Initial Disturbance in general. 26. The Dilatation and the Rotation.-27. Dependence of the Dilatation and the Rotation upon their Initial Values.-28. Dependence of the Displacenent upon Initial Dilatation.29. Dependence of the Displacement upon Initial Rotation.-30. Discontinuities at WaveFronts.-31. Tendency to the Formation of Surfaces of Discontinuity.-32. Conditions to be satisfied at the Initial Boundary. - 33. Application of these Conditions to the Crise of Spherical Waves of Sound.-34. Simplification of the Results by the Conditions at the Initial Boundary. -35. Dilatation and Rotation at the Fronts and Rerrs of Waves.-36. Summary.

1. The investigations contained in this paper originated in the discovery of an oversight in the analysis in Stokes's justly famous memoir on the "Dynamical Theory of Diffraction." To make this orersight apparent it is necessary to describe in some detail the contents of the second section of that memoir. This section deals with the propagation of an arbitrary disturbance in an elastic medium. The medium is taken to be an isotropic elastic solid, and the disturbance is supposed to be confined initially to a portion of it which occupies a finite volume. The dilatation $\Delta$ and the components of the rotation $\varpi$ satisfy respectively the partial differential equations $\partial^{2} \Delta / \partial t^{2}=a^{2} \nabla^{2} \Delta$ and $\hat{\sigma}^{2} \varpi / \partial t^{2}=b^{2} \nabla^{2} \varpi$, and they are calculated from them by means of Poisson's integral formula. $t$ According to this formula any function $f$ which satisfies an

- Cainbridge Phil. Soc. Trans., Vol. Ix., 1849. The memoir is reprinted in Stokes's Mathematical and Physical Papers, Vol It., p. 243 (Cambridge, 1883). The detiled references in the sequel are to the pages of the reprint.

† Given by Poisson, Mém. de l'Institut, Paris, t. III., 1820. 
equation of the form $\partial^{2} f / \partial t^{2}=a^{2} \nabla^{2} f$ can be expressed in terms of initial values by the equation

$$
f=\frac{t}{4 \pi} \iint \dot{f}_{0}(a t) d \sigma+\frac{\partial}{\partial t}\left\{\frac{t}{4 \pi} \iint f_{0}(a t) d \sigma\right\},
$$

in which the integration refers to angular space about the point at which $f$ is estimated, and $f_{0}(a t)$ and $\dot{f}_{0}(a t)$ denote the initial values of $f$ and $\hat{\mathrm{d}} f / \partial t$ on a sphere of radius at with its centre at the point. The components of displacement are calculated from the values obtained for the dilatation and rotation by the intervention of a scalar potential correlated with the dilatation and a vector potential correlated with the rotation. The process of calculation involves considerations of some delicacy. From the expressions obtained for the displacement due to initial disturbance Stokes deduced the effect of force operative at a point.

From this description it will be seen that all Stokes's results depend upon the employment of Poisson's integral formula to express the dilatation and the components of the rotation. In a recent paper* I have pointed out that this formula does not in general yield correct expressions for these quantities. In the same paper I identified the formula (A) with one that has been used by Poincarét and others, viz.,

$$
f=\frac{1}{4 \pi} \iint\left(t \dot{f}_{0}+f_{0}+r \frac{\partial f_{0}}{\partial r}\right)_{r=a t} d \sigma
$$

where $r$ denotes distance from the point at which $f$ is estimated.

The reason for the failure of such formulæ as (B) to represent the dilatation and the components of rotation is clear from an inspection of (B). When the point at which the disturbance is estimated is near the front of an advancing wave the sphere described about the point penetrates but a little way into the region within which the initial disturbance is confined, and the part of the sphere which is included in the integration is very small. Thus the formula cannot express any quantity which has a value different from zero at the front of an advancing wave. Now there is no kinematical or dynamical reason why the dilatation and rotation in an elastic solid should be supposed to vanish at the front of an advancing wave, and it appears therefore that Stokes's analysis is adequate to expross the effects of particular types of initial disturbance, but not those of an arbitrary initial disturbance confined to a finite portion of the medium.

With the object of clearing up the difficulty thus encountered I begin

- Supra, p. 3i.

† Theorie math. de la lumière, t. 1., Paris, 18s9, p. 96. The conditions of identity of (A) and (B) ore copsidered in Article 23 infra. 
by investigating the effects produced by force operative at a point. It is then possible to retrace the steps of Stokes's investigation and to arrive at a solution of the problem of arbitrary initial disturbances. It is also possible to obtain solutions of other problems relating to the propagation of wave-motions through an isotropic elastic solid medium. A summary of the results will be found at the end of the paper.

2. Notation.-The following notation will be used :-

$\rho$ denotes the density of the medium.

$\lambda$ and $\mu$ are the elastic constants of Lamé ; so that $\mu$ is the modulus of rigidity and $\lambda+\frac{2}{3} \mu$ is the modulus of compression.

$a$ and $b$ are the velocities of irrotational and equivoluminal waves; so that $a^{2}=(\lambda+2 \mu) / \rho, b^{2}=\mu / \rho$.

$(u, v, w)$ is the displacement at any point $(x, y, z)$ at time $t$.

$\Delta$ is the dilatation; so that $\Delta=\operatorname{div}(u, v, w)=\partial u / \partial x+\partial v / \partial y+\partial w / \partial z$.

$\left(\boldsymbol{\varpi}^{\prime}, \boldsymbol{\varpi}^{\prime \prime}, \boldsymbol{\varpi}^{\prime \prime \prime}\right)$ is the rotation ; so that $2\left(\boldsymbol{\varpi}^{\prime}, \boldsymbol{\varpi}^{\prime \prime}, \boldsymbol{\varpi}^{\prime \prime \prime}\right)=$ curl $(u, v, w)$, or $2 \varpi^{\prime}=\partial w / \partial y-\partial v / \partial z$, and so on.

$\phi$ is the scalar potential and $(F, G, H)$ the vector potential; so that $(u, v, w)=-$ (gradient of $\phi)+\operatorname{curl}(F, G, H)$, where $\operatorname{div}(F, G, H)=0$, or $u=-\partial \phi / \partial x+\partial H / \partial y-\partial G / \partial z$, and so on; also $\nabla^{2} \phi=-\Delta, \nabla^{2} F=-2 \sigma^{\prime}$, and so on.

$\nabla^{2}$ stands for the operator $\partial^{2} / \partial x^{2}+\partial^{2} / \partial y^{2}+\partial^{2} / \partial z^{2}$.

$r$ denotes the distance between a point $(x, y, z)$ and a point $\left(x^{\prime}, y^{\prime}, z^{\prime}\right)$. time.

A dot over a letter indicates differentiation with respect to the

3. Conditions to be satisfied at a Wave-Boundary.*-When the displacement vanishes outside a wave the boundary of the wave is a surface $\Sigma$; on one side of $\Sigma$ the displacement $(u, v, v)$ vanishes everywhere; on the other side of $\Sigma$ the components $u, \ldots$ are variable functions of $x, y, z$ and of $t$. Since there can be no discontinuity of displacement without rupture of the material, the values assumed by $u, v, w$ on $\Sigma$ vanish. Let $N$ denote the normal to $\Sigma$ at any point, and $s$ any direction in the tangent plane at the point. Since $u$ vanishes all over $\Sigma$, we must have

$$
\frac{\partial u}{\partial x} \frac{d x}{d s}+\frac{\partial u}{\partial y} \frac{d y}{d s}+\frac{\partial u}{\partial z} \frac{d z}{d s}=0
$$

- Cf. my piper already quoted. 
and it follows that

$$
\frac{\partial u / \partial x}{\cos (x, N)}=\frac{\partial u / \partial y}{\cos (y, N)}=\frac{\partial u / \partial z}{\cos (z, N)}=\frac{\partial u}{\partial N}
$$

Like equations hold with $v$ and $w$ in place of $u$.

Again $\Sigma$ is not stationary, but moves normally to itself with a velocity which is either $a$ or $b$. When it is $a$ the equation $u=0$ represents a surface which advances with velocity $a$, and we may take $N$ to be in the direction in which the surface advances. Then we have

$$
\frac{\lambda_{u}}{\partial t}+a\left\{\frac{\partial \hat{u} u}{\partial x} \cos (x, N)+\frac{\hat{\partial} u}{\partial y} \cos (y, N)+\frac{\partial u}{\partial z} \cos (z, N)\right\}=0,
$$

and it follows from (1) that we must have

$$
\frac{\partial u}{\partial N}=-a^{-1} \frac{\partial u}{\partial t} \text {. }
$$

Like equations hold with $v$ and $w$ in place of $u$. If the surface $\Sigma$ advances with velocity $b$, we must write $b^{-1}$ in place of $a^{-1}$.

Equations (1) are simply kinematical conditions of continuity, but the equations of type (2) are dynamical conditions which connect the velocity near a wave-boundary with the strain in the same neighbourhood. When these equations are satisfied, we may say that the velocity at the boundary is " compensated" by the strain.

In the more general case, where the moving surface $\Sigma$ separates two portions of the medium in which the components of displacement are expressed by different analytical functions, there are kinematical conditions of continuity of displacement and dynamical conditions connecting the differences of velocity with the differences of strain. The surface $\Sigma$ moves normally to itself with a velocity which is either $a$ or $b$. If $u^{\prime}, u^{\prime \prime}$ denote the values of $u$ on the two sides of $\Sigma$, the conditions of continuity are of the type $u^{\prime}=u^{\prime \prime}$ at $\Sigma$, and these lead us to equations of the form

$$
\frac{\partial u^{\prime} / \partial x-\partial u^{\prime \prime} / \partial x}{\cos (x, N)}=\frac{\partial u^{\prime} / \partial y-\partial u^{\prime \prime} / \partial y}{\cos (y, N)}=\frac{\partial u^{\prime} / \partial z-\partial u^{\prime \prime} / \partial z}{\cos (z, N)}=\frac{\partial u^{\prime}}{\partial N}-\frac{\partial u^{\prime \prime}}{\partial N},
$$

and the dynamical conditions lead to additional relations of the type

$$
\frac{\partial u^{\prime}}{\partial N}-\frac{\partial u^{\prime \prime}}{\partial N}=-a^{-1}\left(\frac{\partial u^{\prime}}{\partial t}-\frac{\partial u^{\prime \prime}}{\partial t}\right)
$$

if the surface $\Sigma$ moves with the velocity $a$. If it moves with the velocity $b$, we must write $b^{-1}$ in place of $a^{-1}$.

When a wave with velocity $a$ is propagated, the dilatation and the 
normal component velocity are connected by the equation

$$
\begin{aligned}
-a\left(\Delta^{\prime}-\Delta^{\prime \prime}\right)=\left\{\dot{u}^{\prime} \cos (x, N)\right. & \left.+\dot{v}^{\prime} \cos (y, N)+\dot{v}^{\prime} \cos (z, N)\right\} \\
& -\left\{\dot{u}^{\prime \prime} \cos (x, N)+\dot{v}^{\prime \prime} \cos (y, N)+\dot{v}^{\prime \prime} \cos (z, N)\right\} .
\end{aligned}
$$

When a wave with velocity $b$ is propagated the rotation and the tangential components of velocity are connected by equations of the type

$-b\left\{\left(\frac{\partial w^{\prime}}{\partial y}-\frac{\partial v^{\prime}}{\partial z}\right)-\left(\frac{\partial w^{\prime \prime}}{\partial y}-\frac{\partial v^{\prime \prime}}{\partial z}\right)\right\}$

$$
=\left\{\dot{w}^{\prime} \cos (y, N)-\dot{v}^{\prime} \cos (z, N) ;-\dot{i} \dot{v}^{\prime \prime} \cos (y, N)-\dot{v}^{\prime \prime} \cos (z, N)\right\} .
$$

4. Displacement due to Body Forces.-We consider the case where the motion of the medium is produced and maintained by the action of forces which are operative at the points within a certain region $T$. Let $(X, Y, Z)$ be the body force per unit of mass. The equations of motion can be written in such forms as

$$
(\lambda+\mu) \frac{\partial \Delta}{\partial x}+\mu \nabla^{2} u+\rho X=\rho \frac{\hat{\sigma}^{2} u}{\partial t^{2}} .
$$

If $(X, Y, Z)$ were zero, the solutions of these equations would represent free motions of the medium, such, for example, as can be due to forces which have ceased to act. Solutions representing free motions can be added to any particular solutions of the equations (5), containing $(X, Y, Z)$, and the difference between such particular solutions will always represent a free motion. The effects produced by the forces can therefore be found by obtaining particular solutions of the equations (5).

For this purpose we express the field of force in terms of a scalar potential $\Phi$ and a vector potential $(L, M, N)$, so that

$$
(Y, Y, Z)=- \text { (gradient of } \Phi)+\operatorname{curl}(L, M, N) \text {. }
$$

With notation already introduced, equation (5) then becomes

$$
\begin{aligned}
-(\lambda+2 \mu) \frac{\partial}{\partial x} \nabla^{2} \phi+\mu\left(\frac{\partial}{\partial y} \nabla^{2} H-\frac{\partial}{\partial z} \nabla^{2} G\right)-\rho & \left(\frac{\partial \Phi}{\partial x}-\frac{\partial N}{\partial y}+\frac{\partial M}{\partial z}\right) \\
& =-\rho \frac{\partial^{2}}{\partial t^{2}}\left(\frac{\partial \phi}{\partial x}-\frac{\partial H}{\partial y}+\frac{\partial G}{\partial z}\right),
\end{aligned}
$$

and we have two other equations like this. It is clear that a particular solution will be obtained if we can solve the equations

$$
\frac{\partial^{2} \phi}{\partial t^{2}}-a^{2} \nabla^{2} \phi=\Phi, \quad \frac{\hat{\sigma}^{2} F}{\partial t^{2}}-b^{2} \nabla^{2} F=L, \quad \ldots
$$


It is well known that particular solutions of these equations can be expressed in the forms*

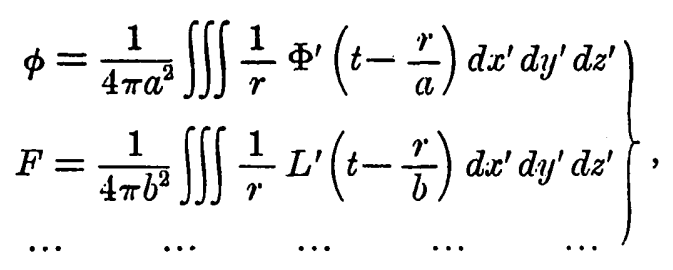

in which $\Phi^{\prime}(t-r / a)$ denotes that the value of $\Phi$ at the point $\left(x^{\prime}, y^{\prime}, z^{\prime}\right)$ is to be calculated at the instant $t-r / a$, with a like signification for $I^{\prime}(t-r / b), \ldots$, and the integration extends through all space in which $\Phi^{\prime}, \ldots$ are different from zero at the times indicated.

Now $\Phi, L, M, N$ can be expressed in the forms

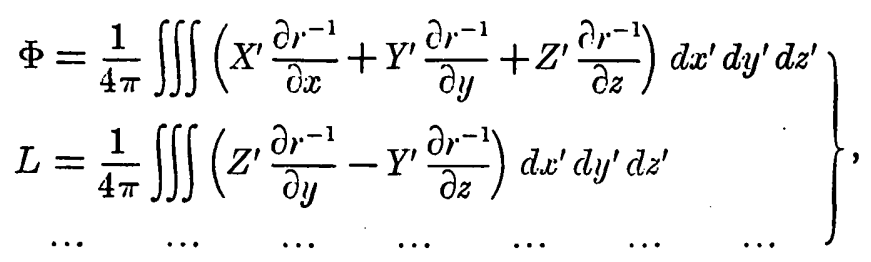

where the integration extends through the region $T$ in which $(X, Y, Z) \neq 0$. In fact, it appears at once that, when these forms are assumed and the gradient of $\Phi$ and the curl of $(L, M, N)$ are calculated from them,

$$
-(\text { gradient of } \Phi)+\operatorname{curl}(L, N, N)=(X, Y, Z) \text { or zero, }
$$

according as the point $(x, y, z)$ is within $T$ or not.

5. Potentials of Disturbance due to Force at a Point.-We may use our results to obtain the effect of force operative at one point. It will be sufficient to consider the case of a force of variable magnitude $\chi(t)$ acting at the point $\left(x^{\prime}, y^{\prime}, z^{\prime}\right)$ in the direction of the axis of $x$. In equations (8) we pass to a limit by supposing all the linear dimensions of the region $T$ to be dininished indefinitely, but the integral of $X^{\prime}$ through this region to remain finite, so that

$$
\iiint \rho X^{\prime} d x^{\prime} d y^{\prime} d z^{\prime}=\chi(t), \quad \iiint \rho Y^{\prime} d x^{\prime} d y^{\prime} d z^{\prime}=0, \quad \ldots .
$$

* L. Lorenz, J. $f$. Math. (Crelle), Bd. IvIII., 1861. The solution of equations of the type (6) in the case of forces which are simple harmonic functions of the time had been discussed by Helmholtz in the memoir "Theorie d. Luftschwingungen in Röhren mit offenen Enden" in the previous volume of the same journal. 
Then we have

$$
\Phi=\frac{1}{4 \pi \rho} \chi(t) \frac{\partial r^{-1}}{\partial x}, \quad L=0, \quad M=\frac{1}{4 \pi \rho} \chi(t) \frac{\partial r^{-1}}{\partial z}, \quad N=-\frac{1}{4 \pi \rho} \chi(t) \frac{\partial r^{-1}}{\partial y} .
$$

We have to deduce the values of $\phi, F, G, H$ by means of equations (7). For this purpose we observe that in (7) $x^{\prime}, y^{\prime}, z^{\prime}$ are the coordinates of a point at which $\Phi, L, M, N$ are not all zero, and $r$ is the distance of this point from $(x, y, z)$; but in (9) $x^{\prime}, y^{\prime}, z^{\prime}$ are the coordinates of the point of application of the force $\chi(t)$, and $r$ denotes the distance of this latter point from $(x, y, z)$. We therefore rewrite $(7)$ in the form

$$
\phi=\frac{1}{4 \pi a^{2}} \iiint \frac{1}{D}[\Phi] d a d \beta d \gamma, \quad F=\frac{1}{4 \pi b^{2}} \iiint \frac{1}{D}\{L\} d a d \beta d \gamma, \ldots,
$$

in which $D$ denotes the distance of the point $(\alpha, \beta, \gamma)$ from $(x, y, z)$, and $[\Phi],\{L\}, \ldots$ denote the values of $\Phi, L, \ldots$ at the point $(\alpha, \beta, \gamma)$ at the instants $t-D / a, t-D / b$; so that we have

$$
\left.\begin{array}{rl}
{[\Phi]=\frac{1}{4 \pi \rho} \times\left(t-\frac{D}{a}\right) \frac{\partial R^{-1}}{\partial \alpha},} & \{L\}=0, \\
\{M\}=\frac{1}{4 \pi \rho} \times\left(t-\frac{D}{b}\right) \frac{\partial R^{-1}}{\partial \gamma}, & \{N\}=-\frac{1}{4 \pi \rho} \times\left(t-\frac{D}{b}\right) \frac{\partial R^{-1}}{\partial \beta}
\end{array}\right\},
$$

where $R$ denotes the distance of the point $(\alpha, \beta, \gamma)$ from the point $\left(x^{\prime}, y^{\prime}, z^{\prime}\right)$ at which the force $\chi(t)$ is applied.

To perform the integrations in (10) we imagine space to be partitioned into thin sheets by means of spherical surfaces having the point $(x, y, z)$ as centre. Let $D$ be the radius of one such sphere, $d S$ an element of its surface. We observe that $\chi(t-D / a)$ and $\chi(t-D / b)$ are constant over $S$, and thus we have

$$
4 \pi \rho \iiint D^{-1}[\Phi] d a d \beta d \gamma=\int_{0}^{\infty} D^{-1} \chi\left(t-\frac{D}{a}\right) d D \iint\left(\frac{\partial R^{-1}}{\partial \alpha}\right) d S,
$$

and like equations involving $L, M, N$. Now $\iint\left(\partial R^{-1} / \partial \alpha\right) d S$ is equal to zero if the point $\left(x^{\prime}, y^{\prime}, z^{\prime}\right)$ is within $S$ (so that $D>r$ ), but it is equal to $4 \pi D^{2}\left(\partial r^{-1} / \partial x\right)$ if this point is outside $S$ (so that $D<r$ ); for it may be conceived to represent a component of attraction at $\left(x^{\prime}, y^{\prime}, z^{\prime}\right)$ of an uniform distribution of surface density on the sphere $S$, and this attraction vanishes at internal points, and at external points it is the same as if the whole distributed mass were concentrated at the centre of the sphere. Thus the upper limit of integration with respect to $D$ may be replaced by 
$r$, and the surface integrations can be performed. Similar considerations apply to $L, M, N$, and we have therefore

$$
\phi=\frac{1}{4 \pi a^{2} \rho} \int_{0}^{r} \chi\left(t-\frac{D}{a}\right) \frac{\partial r^{-1}}{\partial x} D d D, \ldots .
$$

These results can be expressed by the equations

$$
\left.\begin{array}{rlrl}
\phi & =\frac{1}{4 \pi \rho} \frac{\partial r^{-1}}{\partial x} \int_{0}^{r^{\prime}, a} t^{\prime} \chi\left(t-t^{\prime}\right) d t^{\prime}, & F & =0, \\
G & =\frac{1}{4 \pi \rho} \frac{\partial r^{-1}}{\partial z} \int_{0}^{r, b} t^{\prime} \chi\left(t-t^{\prime}\right) d t^{\prime}, & H=-\frac{1}{4 \pi \rho} \frac{\partial r^{-1}}{\partial y} \int_{0}^{r / b} t^{\prime} \chi\left(t-t^{\prime}\right) d t^{\prime}
\end{array}\right\},
$$

6. Effect of Force at a Point.-From the values of $\phi, F, \ldots$ we may deduce those of the components of displacement. The expression for $u$ is simplified by observing that $\nabla^{2} r^{-1}=0$. The integrals are differentiated in accordance with the rules

$$
\frac{\partial}{\partial x} \int_{0}^{r^{\prime} a} t^{\prime} \chi\left(t-t^{\prime}\right) d t^{\prime}=\frac{r}{a} \chi\left(t-\frac{r}{a}\right) \frac{1}{a} \frac{\partial r}{\partial x}, \ldots
$$

The results are expressed by the equations

$$
\begin{aligned}
& u=\frac{1}{4 \pi \rho} \frac{\partial^{2} r^{-1}}{\partial x^{2}} \int_{r_{i}, a}^{r^{\prime}, b} t^{\prime} \chi\left(t-t^{\prime}\right) d t^{\prime} \\
& +\frac{1}{4 \pi \rho b^{2} r} \times\left(t-\frac{r}{b}\right)+\frac{1}{4 \pi \rho r}\left(\frac{\partial r}{\partial x}\right)^{2}\left\{\frac{1}{a^{2}} \times\left(t-\frac{r}{a}\right)-\frac{1}{b^{2}} \times\left(t-\frac{r}{b}\right)\right\} \\
& v=\frac{1}{4 \pi \rho} \frac{\partial^{2} r^{-1}}{\partial x \partial y} \int_{r, a}^{r ; b} t^{\prime} \chi\left(t-t^{\prime}\right) d t^{\prime} \\
& +\frac{1}{4 \pi \rho r} \frac{\partial r}{\partial x} \frac{\partial r}{\partial y}\left\{\frac{1}{a^{2}} \chi\left(t-\frac{r}{a}\right)-\frac{1}{b^{2}} \chi\left(t-\frac{r}{b}\right)\right\} \\
& w=\frac{1}{4 \pi \rho} \frac{\partial^{2} r^{-1}}{\partial x \partial z} \int_{r, i}^{r_{i} b} t^{\prime} \chi\left(t-t^{\prime}\right) d t^{\prime} \\
& +\frac{1}{4 \pi \rho r} \frac{\partial r}{\partial x} \frac{\partial r}{\partial z}\left\{\frac{1}{a^{2}} \times\left(t-\frac{r}{a}\right)-\frac{1}{b^{2}} \times\left(t-\frac{r}{b}\right)\right\}
\end{aligned}
$$

These expressions for the displacement due to force at a point may be identified readily with those obtained by Stokes (p. 278). When $\chi^{(t)}$ is a constant they are equivalent to the expressions given by Lord 
Kelvin* to represent the statical effect of force at a point of an elastic solid body. I have verified also that the corresponding tractions over the surface of a spherical cavity with its centre at the origin of $r$ are statically equivalent to a single force in the direction of the axis of $x$, and that, in the limit when the radius of the cavity is diminished indefinitely, this force is of magnitude $\chi(t)$.

It is convenient to observe that the dilatation and rotation calculated from (14) are given by the equations

$$
\begin{aligned}
\Delta & =\frac{1}{4 \pi a^{2} \rho} \frac{\partial}{\partial x}\left\{\frac{1}{r} \chi\left(t-\frac{r}{a}\right)\right\}, \quad \varpi^{\prime}=0, \\
2 \varpi^{\prime \prime} & \left.=\frac{1}{4 \pi b^{2} \rho} \frac{\partial}{\partial z}\left\{\frac{1}{r} \chi\left(t-\frac{r}{b}\right)\right\}, \quad 2 \varpi^{\prime \prime \prime}=-\frac{1}{4 \pi b^{2} \rho} \frac{\partial}{\partial y}\left\{\frac{1}{r} \chi\left(t-\frac{r}{b}\right)\right\}\right\}
\end{aligned}
$$

The results for forces in the directions of the axes of $y$ and $z$ can be written down by symmetry, and the results for an arbitrary direction of the force can be found by addition. When we wish to emphasize the relation of the expressions (14) to force parallel to the axis of $x$, and to an expression of the form $\chi(t)$ for the magnitude of the force, we shall write them $u_{1}(\chi), v_{1}(\chi), w_{1}(\chi)$, and a similar notation $\Delta_{1}(\chi), \ldots$ can be used in regard to the dilatation and rotation. Suffixes 2 and 3 will indicate in like manner that the forces are parallel to the axes of $y$ and $z$. When $\chi$ is the same for all, we have the relations

$$
w_{2}=v_{3}, \quad u_{3}=v_{1}, \quad v_{1}=u_{2} ;
$$

also we have the relations

$$
\varpi_{2}^{\prime \prime \prime}=-\varpi_{3}^{\prime \prime}, \quad \varpi_{3}^{\prime}=-\varpi_{1}^{\prime \prime \prime}, \quad \varpi_{1}^{\prime \prime}=-\varpi_{2}^{\prime}, \quad \varpi_{1}^{\prime}=\varpi_{2}^{\prime \prime}=\varpi_{3}^{\prime \prime \prime}=0 .
$$

7. Establishment of the State of Strain due to Constant Force at a Point.-A special example of some interest is afforded by the case in which the force begins to act at a particular instant, increases at an uniform rate from zero to a finite value $X$, attained at the end of an interval $\epsilon$, and thereafter remains constant. The force will be taken to act in the

- Sir W. Thomson, Cambridge and Dublin Math. Jotrnal, 1848, or Mathematical and Physical Paper's, Vol. I., p. 97 (Cambridge, 1882). Cf. Thomson and Tait, Natural Philosophy, Part II., p. 279, and J. Boussinesq, Applications des Potentiels, \&c., p. 82 (Paris, 1885). 
direction of the axis of $x$. The function $\chi(t)$ is determined by the equations

$$
\begin{array}{ll}
\chi(t)=0 & \text { when } \quad t<0, \\
\chi(t)=X t / \epsilon & \text { when } \quad \epsilon>t>0, \\
\chi(t)=X & \text { when } t>\epsilon .
\end{array}
$$

The formulæ (14) show that at a distance $r$ from the point of application of the force the displacement vanishes at any instant $t$ which is such that $t<r / a$. In the subsequent motion we have to distinguish the intervals* (i.) when $r / a+\epsilon>t>r / a$, (ii.) when $r / b>t>r / a+\epsilon$, (iii.) when $r / b+\epsilon>t>r / b$, (iv.) when $t>r / b+\epsilon$.

In the first of these intervals, i.e., when $r / a+\epsilon>t>r / a$, we have

$$
\int_{r, a}^{r, b} t^{\prime} \chi\left(t-t^{\prime}\right) d t^{\prime}=X \varepsilon^{-1} \int_{r a}^{t} t^{\prime}\left(t-t^{\prime}\right) d t^{\prime}
$$

and we find, after a little algebraic rearrangement,

$$
\left.\left.\begin{array}{ll}
u=\frac{X}{4 \pi \rho}\left\{\frac{(t-r / a)^{2}(t+2 r / a)}{6 \epsilon}\right. & \frac{\partial^{2} r^{-1}}{\partial x^{2}}+\frac{t-r / a}{a^{2} \epsilon r}\left(\frac{\partial r}{\partial x}\right)^{2}
\end{array}\right\}\right),
$$

with a similar expression for $w$. Thus the displacement at the front $(r=a t)$ of the advancing wave vanishes, and there is no tendency for it to become large in the neighbourhood of this surface when $\epsilon$ is small. The velocity at the front of the wave does not vanish, but the equation $\partial u / \partial t+a \partial u / \partial r=0$ is satisfied at that surface in accordance with the condition (2) of Article 3. Thus a wave having a definite front is generated. During an interval $\epsilon$, beginning at the instant when the wavefront reaches a point, it may be shown by means of (19) that the displacement is derived from a scalar potential $\phi$, so that

$$
u=-\frac{\partial \phi}{\partial x}, \quad \ldots, \quad \phi=-\frac{X}{4 \pi \rho}\left\{\frac{t^{3}}{6 \epsilon} \frac{\partial r^{-1}}{\partial x}+\frac{t}{2 a^{2} \epsilon} \frac{\partial r}{\partial x}-\frac{x}{3 a^{3} \epsilon}\right\} .
$$

Hence the motion is irrotational and the dilatation is given by the equation

$$
\Delta=\frac{X t}{4 \pi \rho a^{2} \epsilon} \frac{\partial r^{-1}}{\partial x} \text {. }
$$

The dilatation does not vanish at the front of the advancing wave, and

- Some modifications would be made in the results if $\epsilon$ is so long that these intervals overlap; but they are of no great interest. 
near this front it tends to become large if $\epsilon$ becomes very small, unless $X$ also is very small.

In the second interval, i.e., when $r / b>t>r / a+\epsilon$, we have

$$
\int_{r / a}^{r / b} t^{\prime} X\left(t-t^{\prime}\right) d t^{\prime}=X \int_{r ; a}^{t-e} t^{\prime} d t^{\prime}+X \varepsilon^{-1} \int_{t-e}^{t} t^{\prime}\left(t-t^{\prime}\right) d t^{\prime}
$$

and we find after a little reduction that the displacement is derived from a scalar potential $\phi$, where

$$
\phi=-\frac{X}{8 \pi \rho}\left\{\left(t^{2}-t_{\epsilon}+\frac{1}{3} \epsilon^{2}\right) \frac{\partial r^{-1}}{\partial x}+\frac{1}{a^{2}} \frac{\partial r}{\partial x}\right\} ;
$$

so that the motion is irrotational and the dilatation is given by the equation

$$
4 \pi \rho a^{2} \Delta=X \frac{\partial r^{-1}}{\partial x}
$$

Hence the state of dilatation associated with the operation of a constant force is established at any point at the end of the interval $\epsilon$, beginning at the instant when the wave-front reaches the point.

In the third interval, i.e., when $r / b+\epsilon>t>r / b$, we have

$$
\int_{r / a}^{r ; b} t^{\prime} \chi\left(t-t^{\prime}\right) d t^{\prime}=X \int_{r / a}^{t-e} t^{\prime} d t^{\prime}+X \epsilon^{-1} \int_{t-e}^{r / b} t^{\prime}\left(t-t^{\prime}\right) d t^{\prime},
$$

and we find after a little reduction that

$$
\begin{aligned}
& u=\frac{X}{4 \pi \rho}\left[\left\{-\frac{(t-r / b)^{2}(t+2 r / b)}{6 \epsilon}+\frac{1}{2} t(t-\epsilon)+\frac{1}{6} \epsilon^{2}-\frac{1}{2} \frac{r^{2}}{a^{2}}\right\} \frac{\partial^{2} r^{-1}}{\partial x^{2}}\right. \\
& \left.+\frac{1}{a^{2} r}\left(\frac{\partial r}{\partial x}\right)^{2}+\frac{1}{b^{2} r} \frac{t-r / b}{\epsilon}\left\{1-\left(\frac{\partial r}{\partial x}\right)^{2}\right\}\right] \\
& v=\frac{X}{4 \pi \rho}\left[\left\{-\frac{(t-r / b)^{2}(t+2 r / b)}{6 \epsilon}+\frac{1}{2} t(t-\epsilon)+\frac{1}{6} \epsilon^{2}-\frac{1}{2} \frac{r^{2}}{a^{2}}\right\} \frac{\partial^{2} r^{-1}}{\partial x \partial y}\right. \\
& \left.\left.+\left(\frac{1}{a^{2} r}-\frac{t-r / b}{b^{2} \epsilon r}\right) \frac{\partial r}{\partial x} \frac{\partial r}{\partial y}\right]\right)
\end{aligned}
$$

with a similar expression for $w$. The displacement is expressible in the form

$$
u=-\frac{\partial \psi}{\partial x}+\frac{X t}{4 \pi b^{2} \rho \epsilon r}, \quad v=-\frac{\partial \psi}{\partial y}, \quad w=-\frac{\partial \psi}{\partial z},
$$

where $\quad \psi=\frac{X}{4 \pi \rho}\left\{\frac{(t-\epsilon)^{3}}{6 \epsilon} \frac{\partial r^{-1}}{\partial x}-\frac{1}{2 a^{2}} \frac{\partial r}{\partial x}+\frac{t}{2 \epsilon b^{2}} \frac{\partial r}{\partial x}+\frac{2 x}{3 \epsilon b^{3}}\right\}$;

so that the dilatation and the rotation are given by the equations $\Delta=\frac{X}{4 \pi \rho a^{2}} \frac{\partial r^{-1}}{\partial x}, \quad \varpi^{\prime}=0, \quad 2 \varpi^{\prime \prime}=\frac{X t}{4 \pi \rho b^{2} \epsilon} \frac{\partial r^{-1}}{\partial z}, \quad 2 \varpi^{\prime \prime \prime}=-\frac{X t}{4 \pi \rho b^{2} \epsilon} \frac{\partial r^{-1}}{\partial y}$. 
We see that the displacement does not tend to become large when $\epsilon$ is small, but that the rotation does; also the dilatation established in the second interval is unaltered.

Subsequent to the instant $t=r / b+\epsilon$, the state associated with the action of a constant force is maintained, that is to say, we have

$$
\begin{gathered}
\left.u=-\frac{X}{8 \pi \rho}\left(\frac{1}{b^{2}}-\frac{1}{a^{2}}\right) \frac{\partial^{2} r}{\partial x^{2}}+\frac{X}{4 \pi b^{2} \rho r}, \quad v=-\frac{X}{8 \pi \rho}\left(\frac{1}{b^{2}}-\frac{1}{a^{2}}\right) \frac{\partial^{3} r}{\partial x \partial y}\right) \\
w=-\frac{X}{8 \pi \rho}\left(\frac{1}{b^{2}}-\frac{1}{a^{2}}\right) \frac{\partial^{2} r}{\partial x \partial z}
\end{gathered}
$$

and the dilatation and the rotation are expressed by the equations

$\Delta=\frac{X}{4 \pi \rho a^{2}} \frac{\partial r^{-1}}{\partial x}, \quad \varpi^{\prime}=0, \quad 2 \varpi^{\prime \prime}=\frac{X}{4 \pi \rho b^{2}} \frac{\partial r^{-1}}{\partial z}, \quad 2{\sigma^{\prime \prime \prime}}^{\prime \prime}=-\frac{X}{4 \pi \rho b^{2}} \frac{\partial r^{-1}}{\partial y}$.

8. The wave generated by the force is characterized by the presence of four surfaces of discontinuity, viz.: (i.) the front of the irrotational wave, (ii.) the rear of the wave by which the dilatation is established, (iii.) the front of the rotational wave, (iv.) the rear of the wave by which the rotation is established. The first two move with the velocity $a$, and the remaining two with the velocity $b$. The displacement is continuous at all these surfaces. I have verified that the conditions of the type (cf. Article 3)

$$
a\left(\frac{\partial u^{\prime}}{\partial r}-\frac{\partial u^{\prime \prime}}{\partial r}\right)+\left(\frac{\partial u^{\prime}}{\partial t}-\frac{\partial u^{\prime \prime}}{\partial t}\right)=0
$$

are satisfied at the rear of the wave by which the dilatation is established, i.e., at the surface $r=a(t-\epsilon)$, and that the conditions of the type

$$
b\left(\frac{\partial u^{\prime}}{\partial r}-\frac{\partial u^{\prime \prime}}{\partial r}\right)+\left(\frac{\partial u^{\prime}}{\partial t}-\frac{\partial u^{\prime \prime}}{\partial t}\right)=0
$$

are satisfied at the front of the rotational wave, and at the rear of the wave by which the rotation is established, i.e., at the surfaces $r=b t$ and $r=b(t-\epsilon)$. Thus the necessary conditions of continuity and dynamical conditions connecting velocity and strain at wave-fronts are all satisfied.

9. Graphic Representation of the Establishment of the Strain due to Constant Force.-The results obtained in the last article may be illustrated by figures. For this purpose I consider points on the line of action of the force (say the "axis"), and on the plane through its point of application at right angles to the axis (say, the "equatorial" plane), and distinguish 
the part of the axis drawn from the point of application in the sense towards which the force acts as in "front" of the point. At points on the axis the displacement is longitudinal, and at points on the equatorial plane it is transverse. I have taken $a=2 b$; so that Poisson's ratio tor the material is $\frac{1}{3}$. The scale of the figures is fixed by the conventional equations

$$
a=20, \quad b=10, \quad \epsilon=1, \quad \frac{X}{4 \pi \rho}=30,000 .
$$

Fig. 1 shows the gradual establishment of the longitudinal displacement at points on the axis. The abscissæ represent distance from the point of application of the force. The ordinates of the curves represent

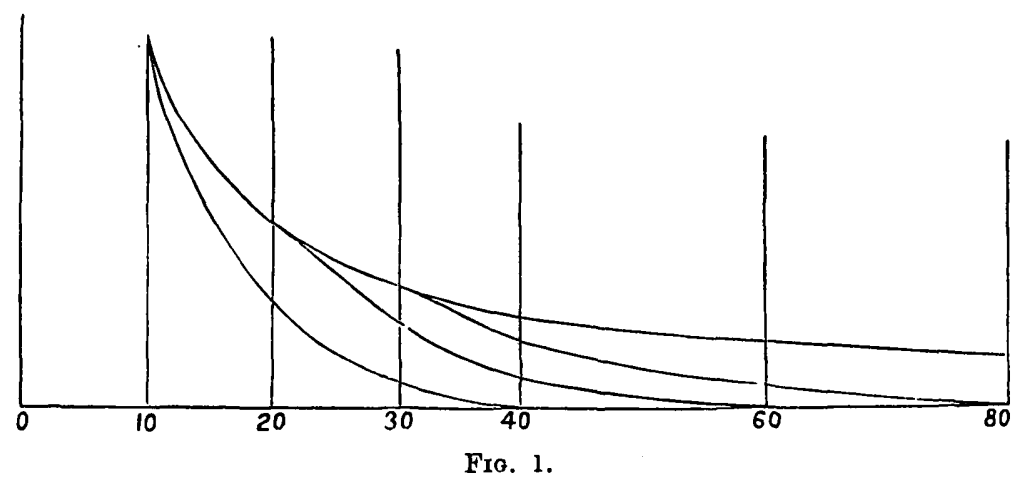

the displacement at definite times. In the uppermost curve the displacement has reached its final value. In the curve which breaks off from this on the ordinate marked 30 the displacement has its value at the instant $t=4$. It is zero beyond $r=80$; the part of the curve between $r=80$ and $r=60$ represents the displacement in the wave by which the dilatation is established; at $r=60$ the direction of the curve changes suddenly ; the part of the curve between $r=40$ and $r=30$ represents the displacement in the wave by which the rotation is established; this curve has an inflexion; behind $r=30$ the ultimate state is estrblished when $t=4$. In the curve which breaks off at the ordinate marked 20 the displacement has its value at the time $t=3$. It is zero beyond $r=60$; the wave by which the dilatation is established lies between $r=60$ and $r=40$, and the wave by which the rotation is established lies between $r=30$ and $r=20$; behind $r=20$ the ultimate state is established when $t=3$. In the curve which breaks off at the ordinate marked 10 the displacement has its value at the instant $t=2$. It is zero beyond $r=40$; the wave by which the dilatation is established lies between $r=40$ and $r=20$, 
and the wave by which the rotation is established lies between $r=20$ and $r=10$; so that there is now no interval between the waves; behind $r=10$ the ultimate state is established when $t=2$.

Fig. 2 shows the transverse displacement at points on the equatorial plane at the time $t=4$. The relative smallness of the displacement in

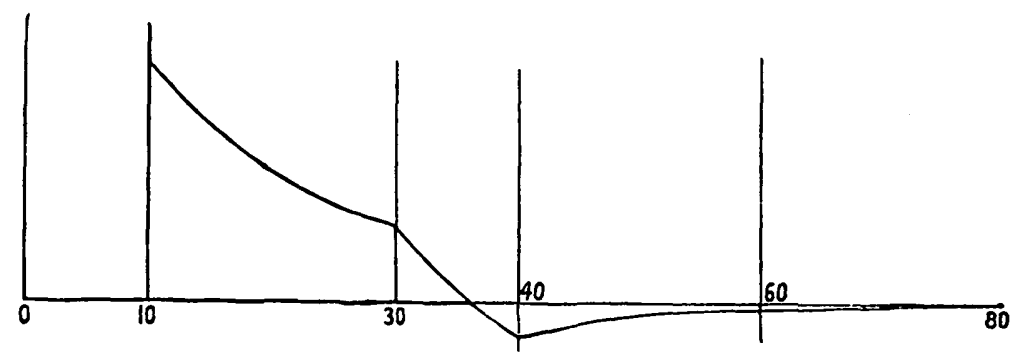

FIg. 2.

the dilatational part of the wave and the result that it is opposite in direction to the force are noteworthy.

Fig. 3 shows the compression at points on the axis and in front of the point of application of the force and the rotation at points in the equatorial

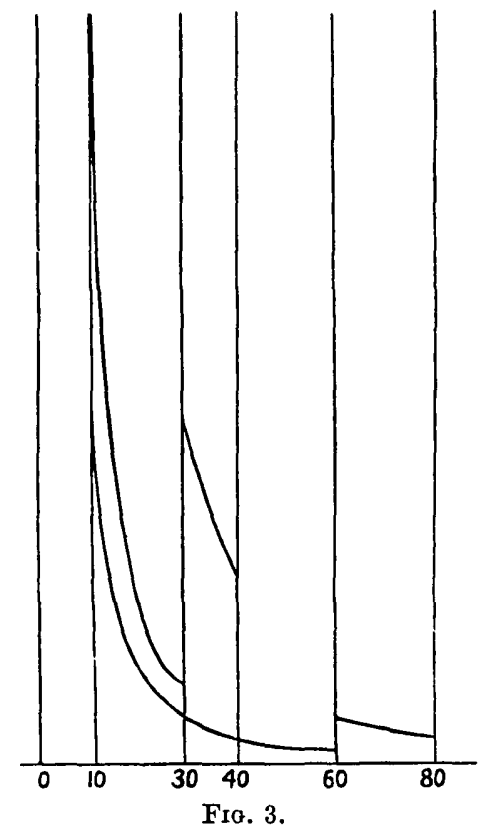

plane, each magnified 50 times from the values given by the conventional scheme of equations (31) adopted for Figs. 1 and 2. The compression is represented by the ordinates of the line which is broken at $r=60$, and 
the rotation by the ordinates of the line which is broken at $r=30$. The dilatation in the part $r<60$ and the rotation in the part $r<30$ are those which belong to the ultimate state.

10. Momentary Force.-We may pass to the example of a force which lasts for a short time by superposing upon the effects due to a force which gradually increases to a certain positive value and then remains constant those due to a force which begins to act a little later than the first, gradually diminishes from zero to a numerically equal negative value, and then remains constant. The dilatation will be confined to a thin pulse of which the front and rear move with velocity $a$, and the rotation to a thin pulse of which the front and rear move with velocity $b$. In front of the pulse of dilatation and behind the pulse of rotation the medium is at rest and without displacement or strain. Between the two pulses there is motion without dilatation or rotation, and therefore also without acceleration; so that this motion is of the type of steady irrotational motion in an incompressible fluid, as was observed by Stokes (p. 279). This motion is that which would be due to a certain doublet at the point of application of the force; the initiation of it involves a relatively intense but momentary state of compression, and the termination of it involves a relatively intense but momentary state of rotational strain.

We may regard a force which acts continually as equivalent to an indefinite succession of small momentary forces. The disturbance produced at any point $O$ at an instant $t$ will consist of (i.) a dilatation or compression corresponding with the initiation of motion due to the force which was operative at the latest instant at which its action can affect the state of the medium at the point $O$ at the instant $t$; (ii.) a rotation corresponding with the termination of motion due to the force which was operative at the earliest instant at which its action can affect the state of the medium at the point $O$ at the instant $t$; (iii.) superposed states of steady motion due to the action of the force at intermediate instants. The combined effect of these latter states may be of equal importance with the dilatation and the rotation (cf. Stokes, p. 279). The result that the magnitude of the force at the intermediate instants affects the state of the medium at a definite point and instant shows that, in some sense, there is propagation of effects through the medium with velocity intermediate between the velocities $a$ and $b$. The recognition of the existence of such effects constitutes the interpretation of the terms of (14) which contain $\int_{r / a}^{r / b} \cdot t^{\prime} \chi\left(t-t^{\prime}\right) d t^{\prime}$, and hence it happens that in the .case of periodic forces of simple harmonic type these effects are entirely masked, for when SEK. 2 . voL. 1 , No. 839 . 
$\chi(t)$ has the form $A e^{\text {ept }}$ the value of the integral is

$$
A\left\{e^{i p(t-r, b)} p^{-2}\left(1+\frac{\imath r p}{b}\right)-e^{i p(t-r ; a)} p^{-2}\left(1+\frac{\imath r p}{a}\right)\right\} ;
$$

so that the whole effect can be analysed into two waves propagated with the velocities $b$ and $a$.

11. Double Forces.-Various types of singular points may be obtained by supposing two or more singularities of the type of points at which forces act to coalesce. The following are the most important types :-

(i.) A force $h^{-1} \chi(t)$ acts at the origin in the positive direction of the axis of $x$, and an equal and opposite force acts at the point $(h, 0,0)$. In the limit, when $h$ is diminished indefinitely while $\chi(t)$ remains the same, the resulting displacement is, in the notation of Article 6,

$$
\left(\frac{\partial u_{1}}{\partial x}, \frac{\partial v_{1}}{\partial x}, \frac{\partial w_{1}}{\partial x}\right)
$$

We may describe the singularity as a "double force without moment." It is related to an axis, in this case the axis of $x$.

(ii.) We may combine three double forces without moment having their axes in the directions of the axes of coordinates. The resulting displacement is

$$
\left(\frac{\partial u_{1}}{\partial x}+\frac{\partial u_{2}}{\partial y}+\frac{\partial u_{3}}{\partial z}, \frac{\partial v_{1}}{\partial x}+\frac{\partial v_{2}}{\partial y}+\frac{\partial v_{3}}{\partial z}, \frac{\partial w_{1}}{\partial x}+\frac{\partial w_{2}}{\partial y}+\frac{\partial w_{3}}{\partial z}\right)
$$

and by equations (16) this is the same as $\left(\Delta_{1}, \Delta_{2}, \Delta_{9}\right)$, or the displacement is

$$
\frac{1}{4 \pi a^{2} \rho}\left(\frac{\partial}{\partial x}, \frac{\partial}{\partial y}, \frac{\partial}{\partial z}\right)\left\{r^{-1} \chi\left(t-\frac{r}{a}\right)\right\} .
$$

The waves that are generated are dilatational waves of a well known type. The singular point may be described as a "centre of dilatation." It has no special relation to any axis. The forces applied in the neighbourhood of this centre have no statical resultant or moment. The state of the medium outside a spherical cavity with its centre at the centre of dilatation could be produced by pressure uniform over the cavity. I find the magnitude of this pressure to be expressed in terms of $\chi$ by the formula

$$
\frac{1}{4 \pi}\left(\nabla^{2}-\frac{4 \mu}{\lambda+2 \mu} \frac{1}{r} \frac{\partial}{\partial r}\right) \frac{\chi(t-r / a)}{r} .
$$

(iii.) We may suppose a force $h^{-1} \chi(t)$ to act at the origin in the positive direction of the axis of $x$, and an equal and opposite force to act at the 
point $(0, h, 0)$, and we may pass to a limit as before. The resulting displacement is

$$
\left(\frac{\partial u_{1}}{\partial y}, \frac{\partial v_{1}}{\partial y}, \frac{\partial w_{1}}{\partial y}\right)
$$

We may describe the singularity as a "double force with moment." The forces applied in the neighbourhood of the singularity are statically equivalent to a couple of moment $\chi(t)$ about the axis of $z$; the singularity is related also to the directions of the forces, i.e., in this case to the axis of $x$.

(iv.) We may combine two double forces with moment, the moments being about the same axis and of the same sign, and the directions of the forces being at right angles to each other. We take the forces to be $h^{-1} \chi(t)$ and $-h^{-1} \chi(t)$ parallel to the axes of $x$ and $y$ at the origin, $-h^{-1} \chi(t)$ parallel to the axis of $x$ at the point $(0, h, 0)$, and $h^{-1} \chi(t)$ parallel to the axis of $y$ at the point $(h, 0,0)$, and we pass to a limit as before. The resulting displacement is

$$
\left(\frac{\partial u_{1}}{\partial y}-\frac{\partial u_{2}}{\partial x}, \frac{\partial v_{1}}{\partial y}-\frac{\partial v_{3}}{\partial x}, \frac{\partial w_{1}}{\partial y}-\frac{\partial w_{2}}{\partial x}\right)
$$

which is the same as $-\mathbf{2}\left(\varpi_{1}^{\prime \prime \prime}, \varpi_{2}^{\prime \prime \prime}, \varpi_{3}^{\prime \prime \prime}\right)$, or it is

$$
\frac{1}{4 \pi b^{2} \rho}\left(\frac{\partial}{\partial y},-\frac{\partial}{\partial x}, 0\right)\left\{r^{-1} \chi\left(t-\frac{r}{b}\right)\right\} \text {. }
$$

The waves that are generated are rotational waves of a well-known type. The forces applied near the singular point are statically equivalent to a couple of moment $2 \chi(t)$ about the axis of $z$. A singular point of this kind may be described as a "centre of rotation about the axis of $z$ "; it is not related to any other axis. The motion expressed by (34) has been discussed with numerical illustrations by Lord Kelvin.*

12. Generalized Double Force.-Other modes of combining double forces are reducible to those already considered, so long as no additional limiting operation is involved. A very general type may be constructed as follows:-Let $\xi$ be any specified component of the traction across a given plane, and let $\xi_{1}, \xi_{2}, \xi_{3}$ be its values when the state of stress is due to force $\chi(t)$ in the directions of $x, y, z$ respectively. Then $\left(\xi_{1}, \xi_{2}, \xi_{9}\right)$ is the displacement due to a certain combination of double forces at the origin.

Denote in general by $X_{x}, Y_{y}, Z_{z}, Y_{z}, Z_{x}, X_{y}$ the six components of stress at a point, and by $X_{v}, Y_{v}, Z_{v}$ the components of traction across the 
plane of which the normal is in the direction $\nu$. Let $l, m, n$ be the direction cosines of $\nu$, so that

$$
X_{\nu}=l X_{x}+m X_{y}+n Z_{x}, \quad Y_{\nu}=l X_{y}+m Y_{y}+n Y_{z}, \quad \ldots ;
$$

also let $\nu^{\prime}$ be the direction in which the traction is to be estimated, and $l^{\prime}, m^{\prime}, n^{\prime}$ the direction cosines of $\nu^{\prime}$; then we have

$$
\begin{aligned}
\xi & =l^{\prime} X_{\nu}+m^{\prime} Y_{\nu}+n^{\prime} Z_{\nu}, \\
\text { or } \xi & =l l^{\prime} X_{x}+m m^{\prime} Y_{y}+n n^{\prime} Z_{z}+\left(m n^{\prime}+m^{\prime} n\right) Y_{z}+\left(n l^{\prime}+n^{\prime} l\right) Z_{x}+\left(l m^{\prime}+l^{\prime} m\right) X_{u} ;
\end{aligned}
$$

so that $\xi$ is related symmetrically to the directions $\nu, \nu^{\prime}$. When the state of stress is due to the action of a force $\chi(t)$ at the origin in the direction of the axis of $x$, the dependence of $\xi$ upon this direction, the directions $\nu, \nu^{\prime}$, and the function $\chi(t)$ may be expressed by writing it $\xi_{1}\left(\nu, \nu^{\prime}, \chi\right)$, and in the same way $\xi_{2}\left(\nu, \nu^{\prime}, \chi\right), \xi_{3}\left(\nu, \nu^{\prime}, \chi\right)$ will denote what $\xi$ becomes when the forces are parallel to the axes of $y$ and $z$, the directions $\nu, \nu^{\prime}$ and the function $\chi$ being the same as before.

Now the stress components are expressed in terms of the strains by equations of the types

$$
X_{x} \doteq \lambda \Delta+2 \mu \frac{\partial \imath}{\partial x}, \quad Y_{z}=\mu\left(\frac{\partial w}{\partial y}+\frac{\partial v}{\partial z}\right) .
$$

The terms of $\xi_{1}, \xi_{2}, \xi_{3}$ that involve $\lambda$ are proportional to $\Delta_{1}, \Delta_{2}, \Delta_{3}$, and are therefore displacements due to a suitable centre of dilatation at the origin. The terms that involve $\partial u_{1} / \partial x, \partial u_{2} / \partial x, \partial u_{3} / \partial x$ may, by equations (16), be replaced by like terms involving $\partial u_{1} / \partial x, \partial v_{1} / \partial x, \partial w_{1} / \partial x$, and they are therefore displacements due to a double force without moment, having for its axis the axis of $x$. The terms that involve $\partial w_{1} / \partial y, \partial w_{2} / \partial y, \partial w_{3} / \partial y$ may be replaced by like terms involving $\partial u_{s} / \partial y, \partial v_{3} / \partial y, \partial w_{3} / \partial y$, and they are therefore displacements due to a double force with moment about the axis of $x$ and having its forces parallel to the axis of $z$. All the remaining terms of $\xi_{1}, \xi_{2}, \xi_{3}$ may be treated in the same way, and thus $\left(\xi_{1}, \xi_{2}, \xi_{3}\right)$ is the displacement due to a certain combination of double forces at the origin.* . A singularity of this kind may be described as a "generalized double force." It is specially and symmetrically related to the pair of directions $\nu, \nu^{\prime}$.

* The result holds of course in the case of equilibrium, i.e., when $\chi(t)$ is replaced by a constant. The result that the quantities expressed by $\xi_{1}, \xi_{2}, \xi_{3}$ when $\chi$ is constant are solutions of the equations which determine the displacement in $a$ body held in equilibrium wris given by C. Somigliuna, Ann. ai mat. (Ser. 2), t. xvr1., 1889. 
13. Multiple Forces.-More complex types of waves of dilatation and rotation may be obtained by repeated differentiations from the formulæ that correspond'with centres of dilatation and centres of rotation. For example, we may put*

$$
(u, v, w)=\left(\frac{\partial}{\partial x}\right)^{a}\left(\frac{\partial}{\partial y}\right)^{\beta}\left(\frac{\partial}{\partial z}\right)^{\gamma}\left(\Delta_{1}, \Delta_{2}, \Delta_{8}\right)
$$

or, again, $\quad(u, v, w)=\left(\frac{\partial}{\partial x}\right)^{a}\left(\frac{\partial}{\partial y}\right)^{\beta}\left(\frac{\partial}{\partial z}\right)^{\gamma}\left(\varpi_{1}^{\prime}, \varpi_{2}^{\prime}, \varpi_{3}^{\prime}\right)$,

and in the latter $\varpi^{\prime}$ may be replaced by $\varpi^{\prime \prime}$ or $\varpi^{\prime \prime \prime}$. All such solutions of the equations of motion can be regarded as derived by suitable limiting operations from combinations of centres of dilatation or of rotation.

An interesting special case is found by combining two pairs of centres of rotation in the same way as two pairs of forces are combined in one such centre. Let there be at the origin centres of rotation with moments $2 h^{-1} \chi(t)$ and $-2 h^{-1} \chi(t)$ about the axes of $x$ and $y$, at the point $(0, h, 0)$ a centre of rotation with moment $-2 h^{-1} \chi(t)$ about an axis parallel to the axis of $x$, and at the point $(h, 0,0)$ a centre of rotation with moment $2 h^{-1} \chi(t)$ about an axis parallel to the axis of $y$, and let us pass to u limit by diminishing $h$ indefinitely. The resulting displacement is

$$
-2 \frac{\partial}{\partial y}\left(\varpi_{1}^{\prime}, \varpi_{2}^{\prime}, \varpi_{3}^{\prime}\right)+2 \frac{\partial}{\partial x}\left(\varpi_{1}^{\prime \prime}, \varpi_{2}^{\prime \prime}, \varpi_{3}^{\prime \prime}\right)
$$

or it is proportional to

$$
\left(\frac{\partial^{2}}{\partial x \partial z}, \frac{\partial^{2}}{\partial y \partial z},-\frac{\partial^{2}}{\partial x^{2}}-\frac{\partial^{2}}{\partial y^{2}}\right)\left\{\frac{1}{r} x\left(t-\frac{r}{b}\right)\right\} .
$$

These are of the same form as the expressions given by Hertzt to represent the electric force in the field around his oscillator. It appears that there has been some discussion $\ddagger$ concerning the nature of the forces required to produce in an elastic solid displacement expressed by the same formulæ.

We may combine two centres of dilatation of opposite signs and very

* These are the types of waves considered by Lord Kelvin in Phil. Mag. (Ser. 5), Vols. xLvIr. and xuviri., 1899.

† Ann. Phys. Chem. (Wiedemann), Bd. xxxvi., 1888, or Electric Waves, English edition, p. 140.

† W. König, Ann. Phys. Chem. (Wiedemann), Bd. xxxvır., 1889 ; and Lord Rayleigh, Theory of Sound, 2nd edition, Vol. Ir., p. 427 (London, 1896), also Phil. Mag. (Ser. 6), Vol. vı., 1903, p. 385 . 
great strength and placed close together on an axis, obtaining a formula of the type

$$
(u, v, w)=\left(\frac{\partial^{2}}{\partial x \partial z}, \frac{\partial^{2}}{\partial y \partial z}, \frac{\partial^{2}}{\partial z^{2}}\right)\left\{\frac{1}{r} \chi\left(t-\frac{r}{a}\right)\right\} .
$$

The states of the medium expressed by (38) and (39) may be supposed to be prodnced by tractions over the surface of a spherical cavity having its centre at the singular point. In both cases the traction is statically equivalent to a single force in the direction of the axis of $z$. Lord Kelvin has discussed these tractions (loc. cit., p. 309), and has shown that the arbitrary functions in (38) and (39) may be adjusted so that the result of superposing the two systems of displacement can represent the effects due to the oscillatory motion of a rigid sphere.

14. Extension of Kirchhoff's Theorem.-In the case of motion determined by a single characteristic equation of the form $\partial^{2} \phi / \partial t^{2}=a^{2} \nabla^{2} \phi$, Kirchhoff* has given a formula of the same kind as Green's formula in the theory of potential, for representing the value of $\phi$ at a point on one side of a closed surface as due to singularities distributed over the surface. By the help of the formulæ for displacement due to force at a point, we can obtain an analogous theorem for the more general case of waves in an elastic solid medium. The method of procedure need not be described in great detail, as the work is very similar to that which I have given elsewhere. $t$ We set out from the reciprocal theorem first given by Betti ${ }_{+}$and expressible in the form

$$
\begin{aligned}
\iiint \rho\left\{\left(X-\frac{\partial^{2} u}{\partial t^{2}}\right) u^{\prime}+\left(Y-\frac{\partial^{2} v}{\partial t^{2}}\right) v^{\prime}\right. & \left.+\left(Z-\frac{\partial^{2} w}{\partial t^{2}}\right) w^{\prime}\right\} d x d y d z \\
& +\iint\left(X_{\nu} u^{\prime}+Y_{\nu} v^{\prime}+Z_{\nu} w^{\prime}\right) d S \\
=\iiint \rho\left\{\left(X^{\prime}-\frac{\partial^{2} u^{\prime}}{\partial t^{2}}\right) u+\left(Y^{\prime}-\frac{\partial^{2} v^{\prime}}{\partial t^{2}}\right) v\right. & \left.+\left(Z^{\prime}-\frac{\partial^{2} w^{\prime}}{\partial t^{2}}\right) w_{1}\right) d x d y d z \\
+ & \iint\left(X_{\nu}^{\prime} u+Y_{\nu}^{\prime} v+Z_{\nu}^{\prime} w\right) d S,
\end{aligned}
$$

in which the surface integration extends over a closed surface $S$, and the volume integration extends through the volume within this surface, and the accented and unaccented letters denote corresponding quantities in two 1891.

- Ann. Phys. Chem. (Wiedemann), Bd. xvrrr., 1883 ; or Vorlesungen ü. math. Optik (Leipzig),

† Phil. Trans. R. S. (Ser. A), Vol. cxovu., 1901.

$\ddagger$ Il nuovo Cimento (Ser. 2), t. vir., 1872. 
states of the medium. Every term in the equation (40) represents the work that would be done by a force of one type acting over a displacement due to force of another type; reversed kinetic reactions are counted as forces in this statement.

We shall suppose that the state $(u, v, w)$ is not due to body force within $S$, and we shall choose for $u^{\prime}, v^{\prime}, w^{\prime}$ the displacement obtained from the $u_{1}, v_{1}, w_{1}$ of Article 6, by replacing $\chi\left(t-t^{\prime}\right)$ by $\chi\left(t+t^{\prime}\right)$ for all values of $t^{\prime}$, the singular point for $u_{1}, v_{1}, v_{1}$ being $\left(x_{0}, y_{0}, z_{0}\right)$. Then $Y^{\prime}$ and $Z^{\prime}$ vanish, and $X^{\prime}$ vanishes except in the immediate neighbourhood of the point $\left(x_{0}, y_{0}, z_{0}\right)$, and we may put

$$
\iiint \rho\left(X^{\prime} u+Y^{\prime} v+Z^{\prime} w\right) d x d y d z=u\left(x_{0}, y_{0}, z_{0}, t\right) \chi(t) .
$$

We choose for $\chi(t)$ a function which vanishes outside a very small range of values including the value $t_{0}$, and there is so great that the integral $\int \chi(t) d t$ over this range is equal to unity. Then we have

$$
\int d t \iint \rho\left(X^{\prime} u+Y^{\prime} v+Z^{\prime} w\right) d x d y d z=u\left(x_{0}, y_{0}, z_{0}, t_{0}\right),
$$

provided that the value $t_{0}$ is included within the range of integration with respect to $t$. We shall write $u^{(0)}$ for $u\left(x_{0}, y_{0}, z_{0}, t_{0}\right)$.

We integrate both members of equation (40) with respect to $t$ over $x$ range of values including $t_{0}$. We may choose the limits of integration so that the integrals of all such terms as $\iiint \rho\left(u \frac{\partial^{2} u^{\prime}}{\partial t^{2}}-u^{\prime} \frac{\partial^{2} u}{\partial t^{2}}\right) d x d y d z$ with respect to $t$ shall vanish, and thus we obtain the formula

$$
u^{(v)}=\iint d S \int_{\iota_{1}}^{\iota_{2}} d t\left(X_{\nu} u^{\prime}+Y_{\nu} v^{\prime}+Z_{\nu} v^{\prime}-X_{\nu}^{\prime} u-Y_{\nu}^{\prime} v-Z_{\nu}^{\prime} v\right),
$$

where $t_{1}$ and $t_{2}$ are suitable extreme values for $t$, and $t_{0}$ lies between them. We may extend the result to space outside a closed surface, or to space bounded by two or more closed surfaces, provided that $u, v, w$ have no singularities in the region of space bounded by the surfaces and containing the point $\left(x_{0}, y_{0}, z_{0}\right)$, and provided that the normal $\nu$ is always drawn out of this region.

15. The integrations with respect to $t$ in the right-hand member of (42) can be performed. Te have

$$
\begin{aligned}
u^{\prime}= & \frac{1}{4 \pi \rho} \frac{\partial^{2} r^{-1}}{\partial x^{2}} \int_{r, i}^{r . b} t^{\prime} \chi\left(t+t^{\prime}\right) d t^{\prime} \\
& +\frac{1}{4 \pi \rho} \frac{1}{r}\left(\frac{\partial r}{\partial x}\right)^{2}\left\lfloor\frac{1}{a^{2}} \chi\left(t+\frac{r}{a}\right)-\frac{1}{b^{2}} \chi\left(t+\frac{r}{b}\right) \biguplus+\frac{1}{4 \pi \rho b^{2} r_{r}} \chi\left(t+\frac{r}{b}\right),\right.
\end{aligned}
$$


and thus integrals of the type $\int_{t_{2}}^{t_{2}} X_{v} u^{\prime} d t$ will consist of two classes of terms, viz., those containing $\int_{r / a}^{r / b} t^{\prime} \chi\left(t+t^{\prime}\right) d t^{\prime}$ and those containing $\chi$. Now $\int_{r / a}^{r / b} t^{\prime} \chi\left(t+t^{\prime}\right) d t^{\prime}$ vanishes unless $t_{0}$ lies between $t+r / a$ and $t+r / b$, and then its value is $\left(t_{0}-t\right)$. It follows that, for any function $F(t)$,

$$
\int_{t_{1}}^{t_{2}} d t F(t) \int_{r / a}^{r / b} t^{\prime} \chi\left(t+t^{\prime}\right) d t^{\prime}=\int_{t_{0}-r / b}^{t_{0}-r / a}\left(t_{0}-t\right) F(t) d t=\int_{r / a}^{r / b} t^{\prime} F\left(t_{0}-t^{\prime}\right) d t^{\prime} .
$$

Again, we have

$$
\int_{t_{1}}^{t_{2}} F(t) \chi\left(t+\frac{r}{a}\right) d t=F\left(t_{0}-\frac{r}{a}\right), \quad \int_{t_{1}}^{t_{2}} F(t) \chi\left(t+\frac{r}{b}\right) d t=F\left(t_{0}-\frac{r}{b}\right) .
$$

Further, it is clear that integrals of the type $\int_{t_{1}}^{t_{2}} X_{v}^{\prime} u d t$ will contain, in addition to terms of the classes already considered, other terms involving $\frac{\partial}{\partial r} \chi\left(t+\frac{r}{a}\right)$ and $\frac{\partial}{\partial r} x\left(t+\frac{r}{b}\right)$. Now we have

$$
\int_{t_{1}}^{t_{2}} F(t) \frac{\partial}{\partial r} \chi\left(t+\frac{r}{a}\right) d t=\frac{1}{a} \int_{t_{1}}^{t_{2}} F(t) \frac{\partial}{\partial t} \chi\left(t+\frac{r}{a}\right) d t,
$$

and this is equal to $-\frac{1}{a} \int_{t_{1}}^{t_{2}} x\left(t+\frac{r}{a}\right) F^{\prime \prime}(t) d t$; or to the value of $-a^{-1} \partial F / \partial t$, when $t=t_{0}-r / a$, and we have therefore

$$
\int_{t_{1}}^{t_{2}} F(t) \frac{\partial}{\partial r} \chi\left(t+\frac{r}{a}\right) d t=\frac{\partial}{\partial r} F\left(t_{0}-\frac{r}{a}\right) .
$$

It follows that the effect of all the integrations with respect to $t$ is to discard $\chi\left(t+t^{\prime}\right)$ and replace $F(t)$ by $F\left(t_{0}-t^{\prime}\right)$ for all values of $t^{\prime}$.

16. The effect of the integration with respect to $t$ in the term $\int_{t_{1}}^{t_{2}} d t \iint X_{\nu} u^{\prime} d S$ is to produce a term $\iint u_{1}\left(X_{\nu}\right) d S$ where $u_{1}\left(X_{\nu}\right)$ means an expression of the form of $u_{1}$ with $X_{\nu}$ in place of $\chi$. Since $u_{1}$ is not altered when the point $(x, y, z)$ and the origin of $r$ are interchanged, the expression $u_{1}\left(X_{\nu}\right) d S$ represents the displacement parallel to the axis of $x$ that would be produced at the point $\left(x_{0}, y_{0}, z_{0}\right)$ by force $X_{v} d S$ acting at $(x, y, z)$ in the 
direction of the axis of $x$. Thus we may write

$$
\int_{t_{1}}^{t_{2}} d t \iint\left(X_{\nu} u^{\prime}+Y_{\nu} v^{\prime}+Z_{\nu} w^{\prime}\right) d S=\iint\left\{u_{1}\left(X_{\nu}\right)+u_{2}\left(Y_{\nu}\right)+u_{3}\left(Z_{\nu}\right)\right\} d S,
$$

and we may interpret this expression as the displacement that would be produced in an unlimited medium by forces acting at the points of $S$, the force at each element of area being equal to the traction exerted across the element in the actual motion.

The effect of the integration with respect to $t$ in the term $-\int_{t_{1}}^{t_{2}} d t \iint X_{\nu}^{\prime} u d S$ is to produce a term $\iint \xi_{1}(x, \nu, u) d S$, where $\dot{\xi}_{1}(x, \nu, u)$ denotes the traction parallel to $x$ across a plane normal to $\nu$ that would be produced at the point $\left(x_{0}, y_{0}, z_{0}\right)$ by a force at $(x, y, z)$, acting in the direction of the axis of $x$, and specified as regards magnitude and dependence on time by $u d S$. The change of sign arises from the observation that, when $(x, y, z)$ and the origin of $r$ are interchanged, the expressions for strain components and stress components that correspond with such displacements as $\left(u_{1}, v_{1}, w_{1}\right)$ are, in fact, changed in sign. Thus we may write

$-\int_{t_{1}}^{t_{9}} d t \iint\left(X_{\nu}^{\prime} u+Y_{\nu}^{\prime} v+Z_{\nu}^{\prime} w\right) d S=\iint\left\{\xi_{1}(x, \nu, u)+\dot{\xi}_{1}(y, \nu, v)+\dot{\xi}_{1}(z, \nu, v)_{j}^{\prime} d S\right.$.

If the direction of $(u, v, w)$ at any point of $S$ were constant as regards time, the right-hand member would take the simpler forn $\iint \xi_{1}(h, \nu, q) d S$, in which $q$ denotes the resultant and $h$ the direction of $(u, v, w)$; but this simplification cannot be made when the ratios $u: v: w$ depend upon $t$, as they generally do.

The final expression for $u^{(0)}$ is

$$
u^{(0)}=\iint\left\{u_{1}\left(X_{v}\right)+u_{2}\left(Y_{\nu}\right)+u_{3}\left(Z_{v}\right)+\xi_{1}(x, \nu, u)+\xi_{2}(y, \nu, v)+\xi_{3}(z, \nu, w)\right\} d S,
$$

and corresponding expressions for $v^{(0)}$ and $w^{(0)}$ can be written down by symmetry.

17. The result expresses the displacement at a point as due to singular points distributed over a surface. The singular points are of two sortspoints at which forces act, and points where there are "generalized double forces." The forces are the actual tractions across the elements of area of the surface. There are three generalized double forces at each point; 
they are related to the three pairs of directions $(x, v),(y, v),(z, v)$, and their mode of dependence upon time is specified by the values of $u, v$, $v$ at the point.

In Kirchhoff's theorem, of which an extension has been given here, the motion is represented as due to sources and doublets. It is not surprising that the singularities which correspond, in the present case, with the sources of the simple theory should be points at which forces are operative, nor that these forces are the actual tractions exerted across the elements of area. It has here been made out that the singularities which, in the present case, correspond with the doublets of the simple theory are the generalized double forces which were investigated in Article 12.

The results are also of interest as emphasizing the result which was found in Article 10, viz., that, in some sense, there are effects which are propagated through the medium with velocities intermediate between $a$. and $b$, as well as others which are propagated with those definite velocities. The effect produced at a point $O$, and at an instant $t$, has been expressed in terms of what was going on at points $P$ of an arbitrary surface, and at instants previous to $t$ by the intervals required to travel from $P$ to $O$ with the velocity $a$, the velocity $b$, and with all intermediate velocities.

18. Disturbance due to an Initial State. - We now take up the problem of determining the effects produced by a state of disturbance given at some instant. At this initial instant we suppose that the displacement and velocity are given at all points within a finite volume $T$, which is bounded by a closed surface, or by two or more closed surfaces. These surfaces will be denoted collectively by $\Sigma_{0}$. We suppose that the displacement and velocity at every point outside $T$ vanish at the initial instant. The degree of arbitrariness in the initial values of the displacement $\left(u_{0}, v_{0}, u_{0}\right)$ and velocity $\left(\dot{u}_{0}, \dot{v}_{0}, \dot{w}_{0}\right)$ is restricted by the conditions which are necessary to secure that there is no rupture of the material. It is therefore clearly necessary that $u_{0}, v_{0}, w_{0}$ vanish at $\Sigma_{0}$. As in Article 3, it follows that, at all points of $\Sigma_{0}$, we must have

$$
\frac{\partial u_{0} / \partial x^{\prime}}{\cos (n, x)}=\frac{\partial u_{0} / \partial y^{\prime}}{\cos (n, y)}=\frac{\partial u_{0} / \partial z^{\prime}}{\cos (n, z)}=\frac{\partial u_{0}}{\partial n},
$$

where $\left(x^{\prime}, y^{\prime}, z^{\prime}\right)$ denotes in general a point within $T$, and $n$ denotes the normal to $\Sigma_{0}$ drawn away from $T$. Similar equations containing $v_{0}$ and $u_{0}$ in place of $u_{0}$ hold also at $\Sigma_{0}$. It will appear later that, to avoid a tendency to rupture of the material, certain relations must connect the components of velocity at points of $\Sigma_{0}$ with the dilatation and the com. 
ponents of rotation at the same points. For the present, howover, we shall proceed as if the initial velocity could be given arbitrarily.

It is convenient to restrict the arbitrariness of the initial displacement and velocity by the conditions that the components of these two vector quantities are expressed by functions of $x^{\prime}, y^{\prime}, z^{\prime}$ which are analytic and without singularities in the region $T$. We may then apply ordinary rules of transformation to definite integrals taken through volumes partly bounded by $\Sigma_{0}$, or over surfaces having edges on $\Sigma_{0}$, without having to. pay attention to any exceptional points other than the points of $\Sigma_{0}$.

Greater generality could be attained, at the expense of greater complication, by supposing that the initial displacement and velocity are expressed by different analytic functions in different parts of $T$. At the surfaces separating such parts the displacement is necessarily continuous, but the velocities and strains are not necessarily continuous. When the velocities and strains are discontinuous in crossing such surfaces, the discontinuities travel through the medium, and the adjustments of velocity and strain described in Article 3 are made in the subsequent motion; difficulty can arise if they are not made initially. An example in which they are made initially would be afforded by the state of the medium produced by a single force, which gradually increases from zero to a definite value and then diminishes again to zero. Any instant after that at which the force ceases to act may be selected as initial instant, and then discontinuities of the kind here described will exist initially, but no tendency to rupture will arise. When the displacements and velocities are expressed by different analytic functions in different parts of $T$, but the velocities and strains are continuous in crossing the surfaces that separate such parts, no tendency to rupture can arise from the initial existence of such surfaces. Wave-motion which involves the continuous existence of such analytic discontinuities will be generated. This class of motions in the case of a fluid medium has been specially studied by H. Hugoniot.* We shall, in general, as already stated, exclude such motions from our theory, but it may be stated in advance that the existence of such analytic discontinuities cannot affect the formulæ (64) which will be obtained for the displacement, though they may affect the formulæ for the dilatation and the rotation.

19. Potentials of Disturbance due to Initial Velocity. - From the effect of force operative at one point we may deduce that due to given

* Jour. de Math. (Liouville), (Ser. 4), tt. 3, 4, 1887, 1888. See also P. Duhem, Recherches sur l' Hydrodynanique, Paris, 1903. 
initial disturbance, in so far as it depends upon initial velocities. We consider, in the first place, an initial velocity $\dot{u}_{0}$, parallel to the axis of $x$, at points of the medium within the volume $T$. The velocity $\dot{u}_{0}$ may be supposed to be communicated to the mass-element $\rho d x^{\prime} d y^{\prime} d z^{\prime}$ by the action, during a very short interval of time $\left(\delta t_{0}\right)$ of a force $X$, directed parallel to the axis of $x$, so that

$$
\rho \dot{u}_{0} d x^{\prime} d y^{\prime} d z^{\prime}=X \delta t_{0}
$$

and we may take the action of $X$ to be confined to the interval between $t=0$ and $t=\delta t_{0}$; so that $X$ vanishes unless $t$ lies between these values. Now, in equations (13) of Article 5 , the integral $\int_{0}^{r / a} t^{\prime} \chi\left(t-t^{\prime}\right) d t^{\prime}$ vanishes if $r<a t$; but, if $r>a t$, it may be replaced by $t X \delta t_{0}$ or by $\rho \dot{u}_{0} t d x^{\prime} d y^{\prime} d z^{\prime}$. It follows that $\dot{u}_{0}$ contributes to $\phi$ the term

$$
(4 \pi)^{-1} \iiint t i u_{0} \frac{\partial r^{-1}}{\partial x} d V_{1}
$$

in which $d V_{1}$ denotes an element of the volume which is within $T$, and is also outside a sphere with centre at $(x, y, z)$ and radius equal to at. We denote this sphere by $S_{1}$, and in like manner we denote by $S_{2}$ the sphere with centre at $(x, y, z)$ and radius equal to $b t$, and by $d V_{2}$ an element of volume outside this sphere and within $T$.

We can now write down the potentials due to initial velocity $\left(\dot{u}_{0}, \dot{v}_{0}, \dot{v}_{0}\right)$ in the forms

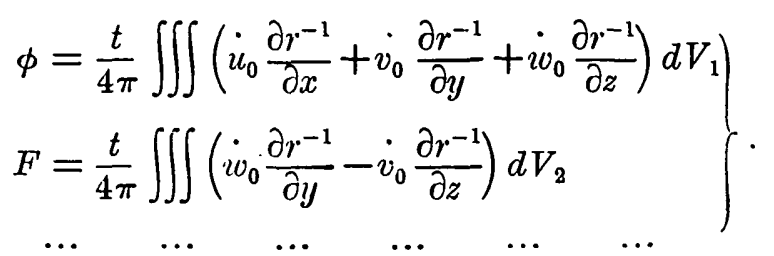

The potentials due to initial displacement are to be obtained by replacing the $\left(\dot{u}_{0}, \dot{v}_{0}, \dot{w}_{0}\right)$ by $\left(u_{0}, v_{0}, w_{0}\right)$ and aifferentiating with respect to $t$. The displacements are derived from the potentials by differentiating with respect to $x, y, z$, and forming certain sums of differential coefficients. In äifferentiating we have to take account of the circumstances that the volumes $V_{1}$ and $V_{2}$ are bounded partly by a fixed surface $\Sigma_{0}$, the boundary of $T$, 
and partly by spheres with centres at $(x, y, z)$ and radii proportional to $t$. We require rules of differentiation adapted to these circumstances.*

20. Rules of Differentiation. - Consider first a volume integral $\iiint \psi d V_{1}$, where $\psi$ involves $x, y, z, t, x^{\prime}, y^{\prime}, z^{\prime}$. If the boundary $S_{1}$ were independent of $x, y, z$ and $t$, we should have

$$
\frac{\partial}{\partial x} \iiint \psi d V_{1}=\iiint \frac{d \psi}{d x} d V_{1}, \quad \ldots, \quad \frac{\partial}{\partial t} \iiint \psi d V_{1}=\iiint \frac{\partial \psi}{\partial t} \partial V_{1} ;
$$

but these require correction because the surface $S_{1}$ depends on $x, y, z, t$. When $(x, y, z)$ is changed into $(x+\delta x, y, z)$, the sphere is shifted from the position $S_{1}$ without altering its radius, and the effect of the shifting is to include in $V_{1}$ an additional volume $-\delta x \iint \frac{x^{\prime}-x}{r} d S_{1}$, where the integration extends over that part of $S_{1}$ which is included in $T$; for $\left\{\left(x^{\prime}-x\right) / r ; \delta\right.$.r is the displacement of a point on the sphere along the normal outwards, and when this is positive at $\left(x^{\prime}, y^{\prime}, z^{\prime}\right)$ an element of volume which was included in $V_{1}$ comes to be excluded. We have therefore

$$
\frac{\partial}{\partial x} \iiint \psi d V_{1}=\iiint \frac{d \psi}{d x} d V_{1}-\iint \frac{x^{\prime}-x}{r} \psi d S_{1} .
$$

Again, when $t$ is changed into $t+\delta t$, the volume $V_{1}$ is diminished by $\iint a \delta t d S_{1}$, and, since $r=a t$ on $S_{1}$, we have

$$
\frac{\partial}{\partial t} \iiint \psi d V_{1}=\iiint \frac{\partial \psi}{\partial t} d V_{1}-\iint \frac{r}{t} \psi d S_{1} \text {. }
$$

Exactly similar formulæ hold with $V_{2}$ and $S_{2}$ substituted for $V_{1}$ and $S_{1}$, the surface integration extending over the portion of $S_{2}$ that is within $T$.

21. Differentiation of Surface Integrals.-The corrections of such formulæ as

$$
\frac{\partial}{\partial x} \iint \psi \partial S_{1}=\iint \frac{\partial \psi}{\partial x} d S_{1} \quad \text { and } \quad \frac{\partial}{\partial t} \iint \psi d S_{1}=\iint \frac{\partial \psi}{\partial t} d S_{1}
$$

are more complicated. When the centre of the sphere of radius at is shifted through $\delta x$, the value of $\psi$ has to be integrated over the surface derived from $S_{1}$ by the shifting, and not over the surface $S_{1}$. Besides

* The expression for $\phi$ is equivalent to that obtained by Stokes, and apparently the values of $F, G, H$ are equivalent to those which he used, but the rules of differentiation are different from those employed by him. 
this, some additional elements of area are included in $T$. These elements adjoin the curve $s_{1}$ in which the surface $S_{1}$ cuts the boundary $\Sigma_{0}$ of $T$. To determine the increment of $\iint \psi d S_{1}$ due to the shifting of the sphere, we express the integral as $\iint \psi_{r} r^{2} d \sigma$, where $d \sigma$ is an element of the solid angle subtended at $(x, y, z)$ by any surface having $s_{1}$ for edge. The element $d \sigma$ is unaltered by the shifting, and any point $P$, associnted with a definite $d \sigma$, is replaced by $\Omega$ new point $Q$ on the same radius rector drawn from $(x, y, z)$, which is the point $O$ in Fig. 4. Now $P Q=\left\{\left(x^{\prime}-x\right) / v\right\} \delta x$, and thus the increment of $\iint \psi d S_{1}$ on account of the shifting of the sphere is

$$
\delta x \iint \frac{x^{\prime}-x}{r} \frac{\partial\left(\psi r^{2}\right)}{\partial r} d \sigma,
$$

or it is $\quad \delta x \iint \frac{x^{\prime}-x}{r^{3}} \frac{\partial\left(\psi r^{2}\right)}{\partial r} d S_{1}$.

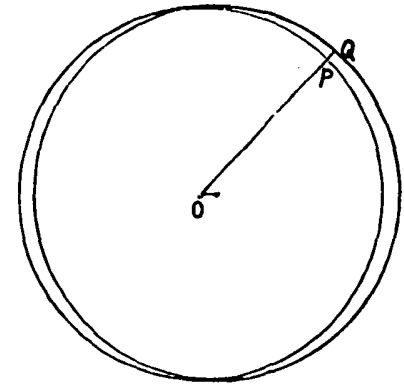

Fro. 4.

Again, when the sphere is shifted through $\delta x$ a strip of a certain width along the curve $s_{1}$ comes to be included in the part of $S_{1}$ which lies within $T$. Let $\epsilon$ denote the width of this strip, $n$ the direction of the outward drawn normal to the boundary $\Sigma_{0}$ of $T$, and let $\chi$ be the angle at which the surfaces $S_{1}$ and $\Sigma_{0}$ cut. The quantity $\epsilon$ would not be altered if we supposed $S_{1}$ to remain fixed and $\Sigma_{0}$ to be shifted through $-\delta x$. The new position of $s_{1}$ could then be obtained from the old by a displacement of magnitude $\epsilon$ directed at right angles to $r$ and to the tangent of $s_{1}$, and the component of this displacement which is in the direction of $n$ can be expressed either as $\epsilon \sin \chi$ or as $-\delta x \cos (x, n)$. Hence the additional area in question is $-\delta x \int \cos (x, n) \operatorname{cosec} \chi d s_{1}$, and we obtain the rule of differentiation

$$
\frac{\partial}{\partial x} \iint \psi d S_{1}=\iint\left\{\frac{\partial \psi}{\partial x}+\frac{x^{\prime}-x}{r^{3}} \frac{\partial}{\partial r}\left(\psi r^{2}\right)\right\} d S_{1}-\int \frac{\cos (x, n)}{\sin \chi} \psi d s_{1} .
$$

The rules for differentiation of $\iint \psi d S_{1}$ with respect to $t$ are of the same kind as the above. When $t$ is changed into $t+\delta t$, we have a correction depending on the change of position of the part of $S_{1}$ that is within $\Sigma_{0}$, and another correction depending on the inclusion of additional elements of area within $\Sigma_{0}$. The first correction is found by taking $\iint \psi d S_{1}$ in the form $\iint \psi r^{2} d \sigma$ to be $\iint a r^{-2}\left\{\partial\left(\psi r r^{2}\right) / \partial r\right\} d S_{1}$. To determine the other correction, let $P$ be any point on $s_{1}$, and let the radius vector $O P$ meet in $Q$ the sphere of radius $a(t+\delta t)$ described about $O$ as centre. In the 
plane containing $P, Q$ and the normal $n$ to the surface $\Sigma_{0}$ at $P$, the sphere of radius $a(t+\delta t)$ will cut $\Sigma_{0}$ in a point $R$ near to $Q$, so that $Q R=-a \delta t \cot (r, n)$, and the increment of $\iint \psi d S_{1}$ on account of the inclusion of elements of area of breadths specified in general by $Q R$ is $-\delta t \iint a \psi \cot (r, n) d s_{1}$. Observing that $\sin (r, n)$ is the same as $\sin \chi$, we have the formula

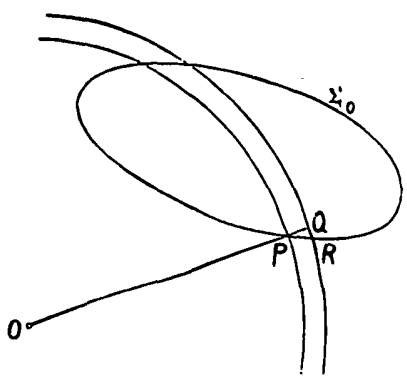

Fir.

$$
\frac{\partial}{\partial t} \iint \psi d S_{1}=\iint\left(\frac{\partial \psi}{\partial t}+\frac{1}{r t} \frac{\partial}{\partial r}\left(\psi r^{2}\right)\right) d d S_{1}-\int a \frac{\cos (r, n)}{\sin \chi} \psi d s_{1} .
$$

Exactly similar rules to those expressed in (56) and (57) hold for the differentiation of surface integrals, such as $\iint \psi d S_{2}$ taken over the part of $S_{2}$ that is within $\Sigma_{0}$. In formulating them the curve of intersection of $S_{2}$ with $\Sigma_{0}$ may be denoted by $s_{2}$.

22. Displacement due to Initial Velocity.-We have now to form expressions for $u, v, w$ from the $\phi, F, G, H$ given in (53), by the formula

$$
(u, v, w)=-(\text { gradient of } \phi)+\operatorname{curl}(F, G, H),
$$

and the rules of differentiation. The contribution of $\dot{u}_{0}$ to $u$ is

$$
\begin{aligned}
& -\frac{t}{4 \pi} \iiint \dot{u}_{0} \frac{\partial^{2} r^{-1}}{\partial x^{2}} d V_{1}-\frac{t}{4 \pi} \iiint \dot{u}_{0}\left(\frac{\partial^{2} r^{-1}}{\partial y^{2}}+\frac{\partial^{2} r^{-1}}{\partial z^{2}}\right) d V_{2} \\
& +\frac{t}{4 \pi} \iint \dot{u}_{0} \frac{x^{\prime}-x}{r} \frac{\partial r^{-1}}{\partial x} d S+\frac{t}{4 \pi} \iint \dot{u}_{0}\left(\frac{y^{\prime}-y}{r} \frac{\partial r^{-1}}{\partial y}+\frac{z^{\prime}-z}{r} \frac{\partial r^{-1}}{\partial z}\right) d S_{2},
\end{aligned}
$$

and this is the same as

$$
\begin{aligned}
\frac{t}{4 \pi} \iiint \dot{u}_{0} \frac{\partial^{2} r^{-1}}{\partial x^{2}} d V+\frac{t}{4 \pi} \iint \dot{u}_{0} \frac{x^{\prime}-x}{r} & \frac{\partial r^{-1}}{\partial x} d S_{1} \\
& +\frac{t}{4 \pi} \iint \dot{u}_{0}\left(\frac{1}{r^{2}}-\frac{x^{\prime}-x}{r} \frac{\partial r^{-1}}{\partial x}\right) d S_{2},
\end{aligned}
$$

where $V$ is that part of the volume between $S_{2}$ and $S_{1}$ which is within $T$, and the surface integrations extend over those parts of $S_{1}$ and $S_{2}$ which are within $T$. The contributions of $\dot{v}_{0}, \dot{w}_{0}$ can be expressed in the same way, and we. 
have therefore, for the part of $u$ which depends on initial velocities, the expression

$$
\begin{aligned}
\frac{t}{4 \pi} \iiint\left(\dot{u}_{0} \frac{\partial^{2} r^{-1}}{\partial x^{2}}+\right. & \left.\dot{v}_{0} \frac{\partial^{2} r^{-1}}{\partial x \partial y}+\dot{w}_{0} \frac{\partial^{2} r^{-1}}{\partial x \partial z}\right) d V \\
& +\frac{t}{4 \pi} \iint \dot{q}_{0} \frac{\partial r^{-1}}{\partial x} d S_{1}+\frac{t}{4 \pi} \iint\left(\dot{u}_{0} \frac{1}{r^{2}}-\dot{q}_{0} \frac{\partial r^{-1}}{\partial x}\right) d S_{2}
\end{aligned}
$$

where $\dot{q}_{0}$ denotes the component of $\left(\dot{u}_{0}, \dot{v}_{0}, \dot{v}_{0}\right)$ in the direction of the radius $r$ drawn from the point $(x, y, z)$ to the point $\left(x^{\prime}, y^{\prime}, z^{\prime}\right)$, so that

$$
\dot{q}_{0}=\left(x^{\prime}-x\right) \dot{u}_{0}+\left(y^{\prime}-y\right) \dot{v}_{0}+\left(z^{\prime}-z\right) \dot{w}_{0} .
$$

The expression (58) is equivalent to that obtained by Stokes (p. 268); it has been obtained here, as by him, from the expressions (53) for the potentials due to initial velocity. The expressions of type (58) may also be obtained from the expressions of type (14) for force operative at a point. In this mode of procedure we should have to replace $\chi(t-r / a)$ by $\rho \dot{u}_{0} d x^{\prime} d y^{\prime} d z^{\prime} / \delta t_{0}$ as in Article 19, and further to replace $d x^{\prime} d y^{\prime} d z^{\prime}$ by $a d S_{1} \delta t_{0}$; we should have to make similar substitutions in the case of $\chi(t-r / b)$. The process is the reverse of that employed by Lord Rayleigh in his Theory of Sound, Vol. II., $\$ 276$.

23. Poisson's Integral Formula.-It may be observed that the process which has been used to obtain the displacement due to initial velocity may be applied to obtain Poisson's integral of the characteristic equation of wave-motion, viz., the equation $\partial^{2} f / \partial t^{2}=a^{2} \nabla^{2} f$. We may treat $f$ as a "generalized displacement," and consider it as due to the operation of "generalized forces" $F_{1}(t)$. At points where the forces are acting we have

$$
\frac{\partial^{2} f}{\partial t^{2}}-a^{2} \nabla^{2} f=F_{1}(t)
$$

and we obtain a particular integral in the form

$$
\left(4 \pi a^{2}\right)^{-1} \iiint r^{-1} F_{1}\left(t-\frac{v}{a}\right) d x^{\prime} d y^{\prime} d z^{\prime} .
$$

Initial generalized velocity $\dot{f}_{0}$ may be thought of as due to generalized forces acting for very short intervals contrining the initial instant, and then, by the method sketched in the last Article, we obtain for the part of $f$ due to $\dot{f}_{0}$ the expression $(4 \pi)^{-1} \iint t \dot{f}_{0} r^{-2} d S_{1}$. The effect of $f_{0}$ may be found by Stokes's rule, and it follows that, if $f$ can always be traced to the operation of generalized forces which produce velocity $\dot{f}$, we must have 


$$
f=\frac{t}{4 \pi} \iint \dot{f}_{0}(a t) d \sigma+\frac{1}{4 \pi} \frac{\partial}{\partial t}\left\{t \iint f_{0}(a t) d \sigma\right\},
$$

as in the formula (A) of Article 1. According to the rule of differentiation (57), the value of $f$ is given by the equation

$$
f=\frac{1}{4 \pi} \iint \frac{1}{r^{2}}\left(t \dot{f}_{0}+f_{0}+r \frac{\partial f_{0}}{\partial r}\right) d S_{1}-\frac{1}{4 \pi} \int \frac{\cos (r, n)}{r \sin \chi} f_{0} d s_{1} .
$$

The line integral in the right-hand member of $(60)$ constitutes a correction of the formula (B), and it appears that the formulæ (A) and (B) of Article 1 are not always equivalent.

One consequence of this result is that Poisson's integral formula (A) can represent a solution of $\partial^{2} f / \partial t^{2}=a^{2} \nabla^{2} f$, which is discontinuous at a wave-boundary, although the formula (B), i.e. the right-hand member of (60) with the line integral omitted, cannot represent any such solution. Nevertheless it remains true that the integral formula in question camnot represent all solutions of the equation, and, in particular, that it does not always yield correct expressions for the dilatation and the components of rotation of an elastic solid medium. We may see this most simply by thinking of the case where initially there is no displacement but some velocity within the region $T$, and the initial velocity within $T$ would be possible in an incompressible fluid moving irrotationally. In this case there is initially neither dilatation nor velocity of dilatation, but there is initial velocity within $T$, of which the normal component does not vanish at the surface $\Sigma_{0}$. It is clear that in the wave-motion that ensues there will be dilatation or compression due to the normal component of the initial velocity at the initial boundary. We shall find later on that the correction of formula (60), i.e., of formula (A), when applied to the dilatation depends upon the normal component of the initial velocity at $\Sigma_{0}$, and that the correction of it when applied to the components of rotation depends upon the components of the initial velocity that are tangential to $\Sigma_{0}$.

When a component of displacement is determined by the characteristic equation, its value is expressed by Poisson's integral formula and the forms (A) and (B) are equivalent; for, if $f_{0}$ is a genuine displacement, it necessarily vanishes at all points of $\Sigma_{0}$. Thus the formula is available for expressing the components of displacement when the whole motion is either irrotational or equivoluminal-a well-known result.

24. Displacement due to Initial Disturbance in general.-The part of the displacement $u$ which depends upon initial displacements can be sEr. 2. vor. 1. No. 840 . 
written down at once in the form

$$
\begin{aligned}
\frac{\partial}{\partial t}\left\{\frac{t}{4 \pi} \iiint\left(u_{0} \frac{\partial^{2} r^{-1}}{\partial x^{2}}+v_{0} \frac{\partial^{2} r^{-1}}{\partial x \partial y}+v_{0} \frac{\partial^{2} r^{-1}}{\partial x \partial z}\right) \partial V\right. \\
\left.+\frac{t}{4 \pi} \iint q_{0} \frac{\partial r^{-1}}{\partial x} d S_{1}+\frac{t}{4 \pi} \iint\left(u_{0} \frac{1}{r^{2}}-q_{0} \frac{\partial r^{-1}}{\partial x}\right) d S_{2}\right\}
\end{aligned}
$$

in which $q_{0}$ denotes the initial displacement in the direction $r$; so that

$$
r q_{0}=\left(x^{\prime}-x\right) u_{0}+\left(y^{\prime}-y\right) v_{0}+\left(z^{\prime}-z\right) w_{0} .
$$

On carrying out the differentiations in accordance with the rules given in Articles 20 and 21, we find for the part of the displacement $u$ due to initial displeicements the expression

$$
\begin{aligned}
& \frac{1}{4 \pi} \iiint\left(u_{0} \frac{\partial^{2} r^{-1}}{\partial x^{2}}+v_{0} \frac{\partial^{2} r^{-1}}{\partial x \partial y}+w_{0} \frac{\partial^{2} r^{-1}}{\partial x \partial z}\right) d V \\
&+\frac{1}{4 \pi} \iint\left\{r\left(u_{0} \frac{\partial^{2} r^{-1}}{\partial x^{2}}+v_{0} \frac{\partial^{2} r^{-1}}{\partial x \partial y}+w_{0} \frac{\partial^{2} r^{-1}}{\partial x \partial z}\right)+\frac{\partial r^{-1}}{\partial x}\left(q_{0}+r \frac{\partial q_{0}}{\partial r}\right)\right\} d S_{1} \\
&-\frac{1}{4 \pi} \iint\left\{r\left(u_{0} \frac{\partial^{2} r^{-1}}{\partial x^{2}}+v_{0} \frac{\partial^{2} r^{-1}}{\partial x \partial y}+w_{0} \frac{\partial^{2} r^{-1}}{\partial x \partial z}\right)+\frac{\partial r^{-1}}{\partial x}\left(q_{0}+r \frac{\partial q_{0}}{\partial r}\right)\right. \\
&\left.-\frac{1}{r^{2}}\left(u_{0}+r \frac{\partial u_{0}}{\partial r}\right)\right\} d S_{2},
\end{aligned}
$$

$$
\text { wherein } \quad r \frac{\partial u_{0}}{\partial r}=\left(x^{\prime}-x\right) \frac{\partial u_{0}}{\partial x^{\prime}}+\left(y^{\prime}-y\right) \frac{\partial u_{0}}{\partial y^{\prime}}+\left(z^{\prime}-z\right) \frac{\partial u_{0}}{\partial z^{\prime}} \text {, }
$$

with a like formula for $\partial q_{0} / \partial r$. No line integrals occur in (62) because $u_{0}, v_{0}, w_{0}$ vanish at all points of $\Sigma_{0}$. The complete expression for $u$ is obtained by adding the expressions (58) and (62) and is the same as that given by Stokes (p. 268). It is convenient to record it in the form

$$
\begin{aligned}
& u= \frac{1}{4 \pi} \iiint\left\{\left(t \dot{u}_{0}+u_{0}\right) \frac{\partial^{2} r^{-1}}{\partial x^{2}}+\left(t \dot{v}_{0}+v_{0}\right) \frac{\partial^{2} r^{-1}}{\partial x \partial y}+\left(t \dot{w}_{0}+w_{0}\right) \frac{\partial^{2} r^{-1}}{\partial x \partial z}\right\} d V \\
&+ \frac{1}{4 \pi} \iint\left\{r\left(u_{0} \frac{\partial^{2} r^{-1}}{\partial x^{2}}+v_{0} \frac{\partial^{2} r^{-1}}{\partial x \partial y}+w_{0} \frac{\partial^{2} r^{-1}}{\partial x \partial z}\right)+\frac{\partial r^{-1}}{\partial x}\left(t \dot{q}_{0}+q_{0}+r \frac{\partial q_{0}}{\partial r}\right)\right\} d S_{1} \\
&-\frac{1}{4 \pi} \iint\left\{r\left(u_{0} \frac{\partial^{2} r^{-1}}{\partial x^{2}}+v_{0} \frac{\partial^{2} r^{-1}}{\partial x \partial y}+w_{0} \frac{\partial^{2} r^{-1}}{\partial x \partial z}\right)+\frac{\partial r^{-1}}{\partial x}\left(t \dot{q}_{0}+q_{0}+r \frac{\partial q_{0}}{\partial r}\right)\right. \\
&\left.-\frac{1}{r^{2}}\left(t \dot{u}_{0}+u_{0}+r \frac{\partial u_{0}}{\partial r}\right)\right\} d S_{2} .
\end{aligned}
$$

The formulæ for $v$ and $w$ can be written down by symmetry. 
25. Expressions for the displacement equivalent, except as regards the independence of $a$ and $b$, to those found by Stokes had been obtained previously by Poisson* and Ostrogradskyt by methods involving the synthesis of particular solutions of simple harmonic type, and the nature of the motion had been the subject of considerable discussion. $\ddagger$ The chief point of debate was concerned with the meaning and degree of importance of the terms expressed by volume integrals. Poisson showed that, at a distance from $T$ which is so great that $T$ may be regarded as little more than a point, the motion breaks up into two wave-motions. In the first of these, propagated with the velocity $a$, the displacement is almost purely longitudinal, i.e., directed along the radius drawn from $T$; in the second, propagated with the velocity $b$, the motion is almost purely transverse. Poisson showed also that, in the same case, the displacement between the two waves is small in comparison with that in either wave. Stokes, taking the dilatation and the components of the rotation to be expressed by particular formulæ, pointed out that the first wave involves dilatation without rotation, and the second rotation without dilatation. The motion between them can involve neither dilatation nor rotation, and is of the character of steady irrotational motion in an incompressible fluid. This last result can be verified at once by means of the formulæ (64). It appears in fact that between the two waves, i.e., when the whole of the region $T$ lies between the spheres $S_{1}$ and $S_{2}$, we may write

$$
\begin{aligned}
(u, v, w)=\text { gradient of } \frac{1}{4 \pi} \iiint\left\{\left(u_{0}+\dot{u}_{0} t\right) \frac{\partial r^{-1}}{\partial x}\right. & +\left(v_{0}+\dot{v}_{0} t\right) \frac{\partial r^{-1}}{\partial y} \\
& \left.+\left(w_{0}+\dot{v}_{0} t\right) \frac{\partial r^{-1}}{\partial z}\right\} d V,
\end{aligned}
$$

where the integration extends through the volume $T$; so that $(u, v, v)$ is derived from a potential which satisfies Laplace's equation and involves $t$ linearly.

In general, it is clear that the terms of (64) which contain volume integrals correspond with the terms of (14) which contain $\int_{v_{i} a}^{r \prime \prime} t^{\prime} \chi\left(t-t^{\prime}\right) d t^{\prime}$; so that they represent the effects which have been described in Articles 10 and 17 as being propagated with velocities intermediate between $a$ and $b$.

- Paris, Mém. de l'Acad., t. x. (1831). An account of Poisson's memoir written by himself is contained in Amnales de Chimie et de Physique, t. xurv. (1830).

+ St. Petersburg, Mém. de l'Acad., t. x. (1831). Ostrogradsky's results are recorded also in a note in Poisson's memoir above cited.

¥ Cf. Todhunter and Pearson, History of the Theory of Elasticity, Vol. I., pp. 627-634 (Cambridge, 1886). 
26. The Dilatation and the Rotation.-Stokes's doctrine of the irrotational and equivoluminal character of the two waves depends upon his assumed formulæ for the dilatation and rotation, but its correctness can be seen at once without assuming these formulæ. We observe in fact that the right-hand members of (53) would be harmonic functions if the boundaries of the volumes of integration were invariable, and it follows that the expressions for $-\nabla^{2} \phi,-\nabla^{2} F, \ldots$, i.e., for $\Delta, 2 \sigma^{\prime}, \ldots$ can contain no volume integral terms. Further, all the terms that can occur in $-\nabla^{2} \phi$ will arise from the variability of the boundary of $V_{1}$, and hence we see that the dilatation will be expressed by integrals taken over $S_{1}$ and $s_{1}$ only. In like manner the rotation will be expressed by integrals taken over $S_{2}$ and $s_{2}$ only. It follows that the dilatation will be confined to the wave that travels with velocity $a$, and the rotation to the wave that travels with velocity $b$.

In forming an expression for $\Delta$ by help of the rules given in Articles 20 and 21, we attend in the first instance to the term of $\phi$ that contanıs $\dot{u}_{0}$. It contributes to $-\partial \phi / \partial x$ the terms

$$
-\frac{t}{4 \pi} \iiint \dot{u}_{0} \frac{\partial^{2} r^{-1}}{\partial x^{2}} d V_{1}+\frac{t}{4 \pi} \iint \frac{\left(x^{\prime}-x\right)^{2}}{r^{4}} \dot{u}_{0} d S_{1},
$$

and to $-\partial \phi / \partial y$ the terms

$$
-\frac{t}{4 \pi} \iiint \dot{u}_{0} \frac{\partial^{2} r^{-1}}{\partial x \partial y} d V_{1}+\frac{t}{4 \pi} \iint \frac{\left(x^{\prime}-x\right)\left(y^{\prime}-y\right)}{r^{4}} \dot{u}_{0} d S_{1},
$$

and it contributes similar terms to $-\partial \hat{\phi} / \partial z$. The terms contributed to $-\partial^{2} \phi / \partial x^{2}$ are

$$
\begin{aligned}
& -\frac{t}{4 \pi} \iiint \dot{u}_{0} \frac{\partial^{3} r^{-1}}{\partial x^{3}} d V_{1} \\
& +\frac{t}{4 \pi} \iint\left[\dot{u}_{0}\left\{\frac{x^{\prime}-x}{r} \frac{\partial^{2} r^{-1}}{\partial x^{2}}+\frac{\partial}{\partial x} \frac{\left(x^{\prime}-x\right)^{2}}{r^{4}}\right\}+\frac{\partial \dot{u}_{0}}{\partial r} \frac{\left(x^{\prime}-x\right)^{9}}{r^{5}}\right] d S_{1} \\
& -\frac{t}{4 \pi} \int \frac{\left(x^{\prime}-x\right)^{2}}{r^{4}} \frac{\cos (x, n)}{\sin \chi} \dot{u}_{0} d s_{1} .
\end{aligned}
$$

The terms contributed to $-\partial^{2} \phi / \partial y^{2}$ are

$$
\begin{aligned}
& -\frac{t}{4 \pi} \iiint \dot{u}_{0} \frac{\partial^{3} r^{-1}}{\partial x \partial y^{2}} d V_{1} \\
& +\frac{t}{4 \pi} \iint\left[\dot{u}_{0}\left\{\frac{y^{\prime}-y}{r} \frac{\partial^{2} r^{-1}}{\partial x \partial y}+\frac{\partial}{\partial y} \frac{\left(x^{\prime}-x\right)\left(y^{\prime}-y\right)}{r^{4}}\right\}+\frac{\partial \dot{u}_{0}}{\partial r} \frac{\left(x^{\prime}-x\right)\left(y^{\prime}-y\right)^{2}}{r^{5}}\right] d S_{1} \\
& -\frac{t}{4 \pi} \int \frac{\left(x^{\prime}-x\right)\left(y^{\prime}-y\right)}{r^{4}} \frac{\cos (y, n)}{\sin \chi} \dot{u}_{0} d s_{1},
\end{aligned}
$$


and similar terms are contributed to $-\partial^{2} \phi / \partial z^{2}$. Hence the contribution of $\dot{u}_{0}$ to $-\nabla^{2} \phi$ is found after a little reduction to be

$$
\frac{t}{4 \pi} \iint \frac{x^{\prime}-x}{r^{4}}\left(\dot{2} \dot{u}_{0}+r \frac{\partial \dot{u}_{0}}{\partial r}\right) d S_{1}-\frac{t}{4 \pi} \int \frac{x^{\prime}-x}{r^{3}} \frac{\cos (r, n)}{\sin \chi} \dot{u}_{0} d s_{1} .
$$

It follows that the part of $\Delta$ which depends upon initial velocities is

$$
\frac{t}{4 \pi} \iint\left(\frac{2}{r^{3}} \dot{q}_{0}+\frac{1}{r^{2}} \frac{\partial \dot{q}_{0}}{\partial r}\right) d S_{1}-\frac{t}{4 \pi} \int \frac{\cos (r, n)}{r^{2} \sin \chi} \dot{q}_{0} d s_{1} .
$$

The part of $\Delta$ which depends upon initial displacements can be obtained by means of Stokes's rule, and it will be found that the complete expression for $\Delta$ is given by the equation

$$
\begin{array}{r}
\Delta=\frac{t}{4 \pi} \iint\left(\frac{2}{r^{3}} \dot{q}_{0}+\frac{1}{r^{2}} \frac{\partial \dot{q}_{0}}{\partial r}\right) d S_{1} \\
+\frac{1}{4 \pi} \iint\left(\frac{1}{r} \frac{\partial^{2} q_{0}}{\partial r^{2}}+\frac{3}{r^{2}} \frac{\partial q_{0}}{\partial r}\right) d S_{1} \\
-\frac{1}{4 \pi} \int \frac{\cos (r, n)}{r \sin \chi}\left(\frac{t \dot{q}_{0}}{r}+\frac{\partial q_{0}}{\partial r}\right) d s_{1}
\end{array}
$$

By precisely similar processes it may be shown that the components of rotation are expressed by equations of the type

$$
\begin{aligned}
2 \boldsymbol{\sigma}^{\prime}= & \frac{t}{4 \pi} \iint\left\{\frac{y^{\prime}-y}{r^{3}}\left(\frac{\partial \dot{w}_{0}}{\partial r}+\frac{2 \dot{w}_{0}}{r}\right)-\frac{z^{\prime}-z}{r^{3}}\left(\frac{\partial \dot{v}_{0}}{\partial r}+\frac{\dot{v}_{0}}{r}\right)\right\} d S_{2} \\
& +\frac{1}{4 \pi} \iint\left\{\frac{y^{\prime}-y}{r^{2}}\left(\frac{\partial^{2} w_{0}}{\partial r^{2}}+\frac{3}{r} \frac{\partial w_{0}}{\partial r}\right)-\frac{z^{\prime}-z}{r^{2}}\left(\frac{\partial^{2} v_{0}}{\partial r^{2}}+\frac{3}{r} \frac{\partial v_{0}}{\partial r}\right)\right\} d S_{2} \\
& -\frac{1}{4 \pi} \int \frac{\cos (r, n)}{r \sin \chi}\left\{\frac{y^{\prime}-y}{r}\left(\frac{\dot{t w_{0}}}{r}+\frac{\partial w_{0}}{\partial r}\right)-\frac{z^{\prime}-z}{r}\left(\frac{\dot{t v_{0}}}{r}+\frac{\partial v_{0}}{\partial r}\right)\right\} d s_{2} .
\end{aligned}
$$

But the question of the reduction of the formulæ here obtained for the dilatation and the rotation to the forms assumed by Stokes requires a further examination.

27. Dependence of the Dilatation and Rotation upon their Initial Values.-To examine this question, we transform the line integrals in (67) and (68). For this purpose, we observe that the direction cosines $d x^{\prime} / d s_{1}, \ldots$ 
of the tangent to $s_{1}$ are given by equations of the type

$$
\frac{d x^{\prime}}{d s_{1}}=\frac{1}{\sin \chi}\left\{\frac{y^{\prime}-y}{r} \cos (z, n)-\frac{z^{\prime}-z}{r} \cos (y, n)\right\},
$$

from which we obtain three equations of the type

$$
\cos (r, n) \frac{x^{\prime}-x}{r}-\cos (x, n)=\sin \chi\left(\frac{y^{\prime}-y}{r} \frac{d z^{\prime}}{d s_{1}}-\frac{z^{\prime}-z}{r} \frac{d y^{\prime}}{d s_{1}}\right) .
$$

From these we deduce the equation

$$
\begin{aligned}
\cos (r, n) \dot{q}_{0}= & \dot{u}_{0} \cos (x, n)+\dot{v}_{0} \cos (y, n)+\dot{w}_{0} \cos (z, n) \\
& +\sin \chi\left\{\frac{d x^{\prime}}{d s_{1}}\left(\dot{v}_{0} \frac{z^{\prime}-z}{r}-\dot{w}_{0} \frac{y^{\prime}-y}{r}\right)+\frac{d y^{\prime}}{d s_{1}}\left(\dot{w}_{0} \frac{x^{\prime}-x}{r}-\dot{u}_{0} \frac{z^{\prime}-z}{r}\right)\right. \\
& \left.+\frac{d z^{\prime}}{d s_{1}}\left(\dot{u}_{0} \frac{y^{\prime}-y}{r}-\dot{v}_{0} \frac{x^{\prime}-x}{r}\right)\right\},
\end{aligned}
$$

and hence we find, since $r$ is constant along $s_{1}$ and over $S_{1}$,

$$
\begin{aligned}
\int \frac{\cos (r, n)}{r \sin \chi} \frac{t \dot{q}_{0}}{r} d s_{1}= & t \int\left\{\dot{u}_{0} \cos (x, n)+\dot{v}_{0} \cos (y, n)+\dot{w}_{0} \cos (z, n)\right\} \frac{d s_{1}}{r^{2} \sin \chi} \\
& +t \iint \frac{1}{r^{2}}\left[\frac { x ^ { \prime } - x } { r } \left\{\frac{\partial}{\partial y^{\prime}}\left(\dot{u}_{0} \frac{y^{\prime}-y}{r}-\dot{v}_{0} \frac{x^{\prime}-x}{r}\right)\right.\right. \\
& \left.\left.-\frac{\partial}{\partial z^{\prime}}\left(\dot{w}_{0} \frac{x^{\prime}-x}{r}-\dot{u}_{0} \frac{z^{\prime}-z}{r}\right)\right\}+ \text { two similar terms }\right] d S_{1},
\end{aligned}
$$

where the surfaee integration extends over the part of the surface $S_{1}$ which is within the curve $s_{1}$, i.e., over the part which is within $T$.

The surface integral in (72) is found, after some algebraic rearrangement, to be equal to

$$
\iint\left(\frac{2}{r^{3}} \dot{q}_{0}+\frac{1}{r^{2}} \frac{\partial \dot{q}_{0}}{\partial r}-\frac{1}{r^{2}} \dot{\Delta}_{0}\right) d S_{1}
$$

The line integral $\int \frac{\cos (r, n)}{r \sin \chi} \frac{\partial q_{n}}{\partial r} d s_{1}$ in (67) may be transformed in the same way, and it will be found finally that the expression (67) for $\Delta$ can be written

$$
\begin{aligned}
\Delta= & \frac{1}{4 \pi} \iint \frac{1}{r^{2}}\left(t \dot{\Delta}_{0}+\Delta_{0}+r \frac{\partial \Delta_{0}}{\partial r}\right) d S_{1} \\
-\frac{1}{4 \pi} \int\left\{\left(\frac{t \dot{u}_{0}}{r}+\frac{\partial u_{0}}{\partial r}\right) \cos (x, n)\right. & +\left(\frac{\dot{t v_{0}}}{r}+\frac{\partial v_{0}}{\partial r}\right) \cos (y, n) \\
& \left.+\left(\frac{t \dot{w}_{0}}{r}+\frac{\partial w_{0}}{\partial r}\right) \cos (z, n)\right\} \frac{d s_{1}}{r \sin \chi} .
\end{aligned}
$$


Exactly similar transformations can be effected in the expression (68) for $2 \varpi^{\prime}$, and the result will be found to be

$$
\begin{aligned}
2 \varpi^{\prime}= & \frac{1}{2 \pi} \iint \frac{1}{r^{2}}\left(\dot{t}_{0}^{\prime}+\varpi_{0}^{\prime}+r \frac{\partial \varpi_{0}^{\prime}}{\partial r}\right) d S_{2} \\
& -\frac{1}{4 \pi} \int\left\{\left(\frac{t \dot{w}_{0}}{r}+\frac{\partial w_{0}}{\partial r}\right) \cos (y, n)-\left(\frac{\dot{t v}_{0}}{r}+\frac{\partial v_{0}}{\partial r}\right) \cos (z, n)\right\} \frac{d s_{3}}{r \sin \chi} .
\end{aligned}
$$

Similar expressions can be obtained for $2 \sigma^{\prime \prime}$ and $2 \sigma^{\prime \prime \prime}$. The terms of (73) and (74) which are expressed by line integrals admit of a further transformation by the aid of the relations (50). Taking the terms in the line integral in (73) which contain $\partial u_{0} / \partial r$, we observe that

$$
\begin{aligned}
\frac{\partial u_{0}}{\partial r} \cos (x, n)= & \left(\frac{x^{\prime}-x}{r} \frac{\partial u_{0}}{\partial x^{\prime}}+\frac{y^{\prime}-y}{r} \frac{\partial u_{0}}{\partial y^{\prime}}+\frac{z^{\prime}-z}{r} \frac{\partial u_{0}}{\partial z^{\prime}}\right) \cos (x, n) \\
=\frac{\partial u_{0}}{\partial x^{\prime}} \cos (r, n) & +\frac{y^{\prime}-y}{r}\left\{\frac{\partial u_{0}}{\partial y^{\prime}} \cos (x, n)-\frac{\partial u_{0}}{\partial x^{\prime}} \cos (y, n)\right\} \\
& +\frac{z^{\prime}-z}{r}\left\{\frac{\partial u_{0}}{\partial z^{\prime}} \cos (x, n)-\frac{\partial u_{0}}{\partial x^{\prime}} \cos (z, n)\right\},
\end{aligned}
$$

and the last two terms in the right-hand member can be omitted, since they vanish at all points of $\Sigma_{0}$, and therefore at all points of $s_{1}$. Hence the terms of (73) which depend on the initial strain near to $\Sigma_{0}$ can be expressed in the form $-\frac{1}{4 \pi} \int \Delta_{0} \cos (r, n) \frac{d s_{1}}{r \sin \chi}$. In like manner, taking the terms of the line integral in (74) which involve $\partial w_{0} / \partial r$ and $\partial v_{0} / \partial r$, we may prove that at all points of $s_{2}$

$$
\frac{\partial w_{0}}{\partial r} \cos (y, n)-\frac{\partial v_{0}}{\partial r} \cos (z, n)=\left(\frac{\partial w_{0}}{\partial y^{\prime}}-\frac{\partial v_{0}}{\partial z^{\prime}}\right) \cos (r, n),
$$

and therefore the terms of (74) which depend on the initial strain near to $\Sigma_{0}$ can be expressed in the form $-\frac{1}{2 \pi} \int \varpi_{0}^{\prime} \cos (r, n) \frac{d s_{2}}{r \sin \chi}$.

We may now write the formulæ for $\Delta$ and $2 \varpi^{\prime}$ in the forms

$$
\begin{aligned}
\Delta= & \frac{1}{4 \pi} \iint \frac{1}{r^{2}}\left(t \dot{\Delta}_{0}+\Delta_{0}+r \frac{\partial \Delta_{0}}{\partial r}\right) d S_{1}-\frac{1}{4 \pi} \int \frac{\cos (r, n)}{r \sin \chi} \Delta_{0} d s_{1} \\
& -\frac{t}{4 \pi} \int\left\{\dot{u}_{0} \cos (x, n)+\dot{v} \cos (y, n)+\dot{w}_{0} \cos (z, n)\right\} \frac{d s_{1}}{r^{2} \sin \chi} \\
2 \varpi^{\prime}= & \frac{1}{2 \pi} \iint \frac{1}{r^{2}}\left(t \dot{\varpi}_{0}^{\prime}+\varpi_{0}^{\prime}+r \frac{\partial \varpi_{0}^{\prime}}{\partial r}\right) d S_{2}-\frac{1}{2 \pi} \int \frac{\cos (r, n)}{r \sin \chi}{\varpi_{0}^{\prime} d s_{2}} \\
& -\frac{t}{4 \pi} \int\left\{\dot{w}_{0} \cos (y, n)-\dot{v}_{0} \cos (z, n)\right\} \frac{d s_{2}}{r^{2} \sin \chi} .
\end{aligned}
$$


It appears from these results that the values assumed by Stokes for the dilatation and the components of rotation do, in fact, require correction whenever there is initial velocity at the surface $\Sigma_{0}$. The first line of (75) is the value that would be given for the dilatation by using Poisson's integral formula ; for it is precisely of the same form as equation (60), and and the like holds for (76). The corrections that must be made in Stokes's values are expressed by the second lines of (75) and (76). The correction of the expression for the dilatation depends exclusively upon the normal component of the initial velocity at $\Sigma_{0}$, and the correction of the expression for the rotation depends exclusively upon the components of the initial velocity tangential to $\Sigma_{0}$, as was stated by anticipation in Article 23 .

28. Dependence of the Displacement upon Initial Dilatation.-The dependence of the motion in the waves of dilatation and rotation upon initial conditions can be shown still more explicitly by transforming the expressions for the components of displacement. The volume integral term of (58) may be written

$$
\frac{t}{4 \pi} \iiint\left(\dot{u}_{0} \frac{\partial^{2} r^{-1}}{\partial \dot{x}^{\prime 2}}+\dot{v}_{0} \frac{\partial^{2} r^{-1}}{\partial x^{\prime} \partial y^{\prime}}+\dot{w}_{0} \frac{\partial^{2} r^{-1}}{\partial x^{\prime} \partial z^{\prime}}\right) d V
$$

and this is equal to

$-\frac{t}{4 \pi} \iiint\left\{\frac{\partial}{\partial x^{\prime}}\left(\dot{u}_{0} \frac{x^{\prime}-x}{r^{3}}\right)+\frac{\partial}{\partial y^{\prime}}\left(\dot{v}_{0} \frac{x^{\prime}-x}{r^{3}}\right)+\frac{\partial}{\partial z^{\prime}}\left(\dot{w}_{0} \frac{x^{\prime}-x}{r^{3}}\right)-\frac{x^{\prime}-x}{r^{3}} \dot{\Delta}_{0}\right\} d V$,

which may be transformed into

$$
\begin{aligned}
& \quad \frac{t}{4 \pi} \iiint \frac{x^{\prime}-x}{r^{3}} \dot{\Delta}_{0} d V \\
& -\frac{t}{4 \pi} \iint \frac{x^{\prime}-x}{r^{3}}\left\{\dot{u}_{0} \cos (x, n)+\dot{v}_{0} \cos (y, n)+\dot{w}_{0} \cos (z, n)\right\} d \Sigma_{0} \\
& -\frac{t}{4 \pi} \iint \frac{x^{\prime}-x}{r^{3}} \dot{q}_{0} d S_{1}+\frac{t}{4 \pi} \iint \frac{x^{\prime}-x}{r^{3}} \dot{q}_{0} d S_{2},
\end{aligned}
$$

where the integration expressed by $\iint \ldots d \Sigma_{0}$ extends over that part of $\Sigma_{0}$ which lies between $S_{1}$ and $S_{2}$. The volume integral in (62) may in like manner be transformed into

$$
\frac{1}{4 \pi} \iiint \frac{x^{\prime}-x}{r^{3}} \Delta_{0} d V-\frac{1}{4 \pi} \iint \frac{x^{\prime}-x}{r^{3}} q_{0} d S_{1}+\frac{1}{4 \pi} \iint \frac{x^{\prime}-x}{r^{3}} q_{0} d S_{2},
$$

where there is no $\iint \ldots d \Sigma_{0}$ because $u_{0}, v_{0}, w_{0}$ vanish at $\Sigma_{0}$. 
The surface integrals in (62) also admit of transformation. We have

$$
\begin{aligned}
u_{0} \frac{\partial^{2} r^{-1}}{\partial x^{2}} & +v_{0} \frac{\partial^{2} r^{-1}}{\partial x \partial y}+w_{0} \frac{\partial^{2} r^{-1}}{\partial x \partial z}+\frac{\partial r^{-1}}{\partial x} \frac{\partial q_{0}}{\partial r} \\
& =-\frac{\partial}{\partial x^{\prime}}\left(\frac{g_{0}}{r^{2}}\right)+\frac{x^{\prime}-x}{r^{3}} \frac{\partial q_{0}}{\partial r}+\frac{x^{\prime}-x}{r^{3}} \frac{\partial u_{0}}{\partial x^{\prime}}+\frac{y^{\prime}-y}{r^{3}} \frac{\partial v_{0}}{\partial x^{\prime}}+\frac{z^{\prime}-z}{r^{3}} \frac{\partial w_{0}}{\partial x^{\prime}},
\end{aligned}
$$

which is equal to

$$
\begin{aligned}
-\frac{\partial}{\partial x^{\prime}}\left(\frac{q_{0}}{r^{2}}\right)+\frac{x^{\prime}-x}{r^{3}} \frac{\partial q_{0}}{\partial r}+\frac{x^{\prime}-x}{r^{3}} \Delta_{0} & +\left(\frac{y^{\prime}-y}{r^{3}} \frac{\partial v_{0}}{\partial x^{\prime}}-\frac{x^{\prime}-x}{r^{3}} \frac{\partial v_{0}}{\partial y^{\prime}}\right) \\
& +\left(\frac{z^{\prime}-z}{r^{3}} \frac{\partial w_{0}}{\partial x^{\prime}}-\frac{x^{\prime}-x}{r^{3}} \frac{\partial w_{0}}{\partial z^{\prime}}\right),
\end{aligned}
$$

and this becomes after some reduction

$$
\begin{aligned}
\frac{x^{\prime}-x}{r^{3}} \Delta_{0}+\frac{1}{r^{2}}\{ & -\frac{x^{\prime}-x}{r}\left(\frac{\partial v_{0}}{\partial y^{\prime}}+\frac{\partial v_{0}}{\partial z^{\prime}}\right)+\frac{y^{\prime}-y}{r} \frac{\partial v_{0}}{\partial x^{\prime}}+\frac{z^{\prime}-z}{r} \frac{\partial w_{0}}{\partial x^{\prime}} ; \\
& +\frac{x^{\prime}-x}{r}\left\{\frac{\partial}{\partial y^{\prime}}\left(\frac{y^{\prime}-y}{r^{3}} q_{0}\right)+\frac{\partial}{\partial z^{\prime}}\left(\frac{z-z^{\prime}}{r^{3}} q_{0}\right)\right\} \\
& -\frac{y^{\prime}-y}{r} \frac{\partial}{\partial x^{\prime}}\left(\frac{y^{\prime}-y}{r^{3}} q_{0}\right)-\frac{z^{\prime}-z}{r} \frac{\partial}{\partial x^{\prime}}\left(\frac{z^{\prime}-z}{r^{3}} q_{0}\right) .
\end{aligned}
$$

Now the integral

$$
\begin{aligned}
\iint \frac{1}{r}\left\{-\frac{x^{\prime}-x}{r}\left(\frac{\partial v_{0}}{\partial y^{\prime}}+\frac{\partial w_{0}}{\partial z^{\prime}}\right)+\frac{y^{\prime}-y}{r} \frac{\partial v_{0}}{\partial x^{\prime}}+\right. & \left.\frac{z^{\prime}-z}{r} \frac{\partial w_{0}}{\partial x^{\prime}}\right\} d S_{1} \\
& =\frac{1}{a t} \int\left(w_{0} \frac{d y^{\prime}}{d s_{1}}-v_{0} \frac{d z^{\prime}}{d s_{1}}\right) d s_{1},
\end{aligned}
$$

which vanishes because $\left(u_{0}, v_{0}, w_{0}\right)$ vanishes along $s_{1}$. In like manner the surface integral of the same quantity taken over $S_{2}$ vanishes, and the integral of the last three terms of (79) when taken over $S_{1}$ or $S_{2}$ also vanishes. Collecting the results, we obtain the equation

$$
\begin{aligned}
u= & \frac{1}{4 \pi} \iiint \frac{x^{\prime}-x}{r^{3}}\left(t \dot{\Delta}_{0}+\Delta_{0}\right) d V+\frac{1}{4 \pi} \iint \frac{x^{\prime}-x}{r^{2}} \Delta_{0} d S_{1}-\frac{1}{4 \pi} \iint \frac{x^{\prime}-x}{r^{2}} \Delta_{0} d S_{2} \\
& -\frac{t}{4 \pi} \iint \frac{x^{\prime}-x}{r^{3}}\left\{\dot{u}_{0} \cos (x, n)+\dot{v}_{0} \cos (y, n)+\dot{w}_{0} \cos (z, n)\right\} d \Sigma_{0} \\
& +\frac{1}{4 \pi} \iint \frac{1}{r^{2}}\left(t \dot{u}+u_{0}+r \frac{\partial u_{0}}{\partial r}\right) d S_{2} .
\end{aligned}
$$

This equation shows the dependence of the motion in the dilatational wave, and between the two waves, upon the initial dilatation and velocity 
of dilatation, and upon the normal component of the initial velocity at the boundary $\Sigma_{0}$ of $T$. The equation shows also that when there is initially no dilatation or velocity of dilatation, and when the normal component of the initial velocity at the surface $\Sigma_{0}$ vanishes, the rotational wave alone is propagated, and the displacement in it is expressed in terms of the initial displacement and velocity by means of Poisson's integral formula, as we know it ought to be. In this case the velocity with which the boundary of the disturbed portion of the medium advances is the velocity $b$.

29. Dependence of the Displacement upon Initial Rotation.-To obtain like results involving the initial rotation, we write

$$
\dot{u}_{0} \frac{\partial^{2} r^{-1}}{\partial x^{2}}+\dot{v}_{0} \frac{\partial^{2} r^{-1}}{\partial x \partial y}+\dot{w}_{0} \frac{\partial^{3} r^{-1}}{\partial x \partial z}
$$

in the form $-\frac{\partial}{\partial x^{\prime}}\left(\frac{\dot{q}_{0}}{r^{2}}\right)+\frac{x^{\prime}-x}{r^{3}} \frac{\partial \dot{u}_{0}}{\partial x^{\prime}}+\frac{y^{\prime}-y}{r^{3}} \frac{\partial \dot{v}_{0}}{\partial x^{\prime}}+\frac{z^{\prime}-z}{r^{3}} \frac{\partial \dot{w}_{0}}{\partial x^{\prime}}$,

and observe that this is equal to

$$
\begin{aligned}
-\frac{\partial}{\partial x^{\prime}}\left(\frac{\dot{q}_{0}}{r^{2}}\right)+\frac{\partial}{\partial x^{\prime}}\left(\frac{x^{\prime}-x}{r^{3}} \dot{u}_{0}\right)+\frac{\partial}{\partial y^{\prime}}\left(\frac{y^{\prime}-y}{r^{3}} \dot{u}_{0}\right) & +\frac{\partial}{\partial z^{\prime}}\left(\frac{z^{\prime}-z}{r^{3}} \dot{u}_{0}\right) \\
& +2 \frac{y^{\prime}-y}{r^{3}} \dot{\varpi}_{0}^{\prime \prime \prime}-2 \frac{z^{\prime}-z}{r^{9}} \dot{\varpi}_{0}^{\prime \prime} .
\end{aligned}
$$

A similar result holds when the dots are omitted, and we may therefore transform the volume integral terms of (64) into

$$
\begin{aligned}
& \frac{1}{2 \pi} \iiint\left\{\frac{y^{\prime}-y}{r^{3}}\left(t \dot{\varpi}_{0}^{\prime \prime \prime}+\varpi_{0}^{\prime \prime \prime}\right)-\frac{z^{\prime}-z}{r^{3}}\left(t \dot{\varpi}_{0}^{\prime \prime}+\varpi_{0}^{\prime \prime}\right)\right\} d V \\
& +\frac{1}{4 \pi} \iint\left\{\frac{\dot{u}_{0} t+u_{0}}{r^{2}}-\frac{x^{\prime}-x}{r^{3}}\left(\dot{q}_{0} t+q_{0}\right)\right\} d S_{1} \\
& -\frac{1}{4 \pi} \iint\left\{\frac{\dot{u}_{0} t+u_{0}}{r^{2}}-\frac{x^{\prime}-x}{r^{3}}\left(\dot{q}_{0} t+q_{0}\right)\right\} d S_{2} \\
& +\frac{t}{4 \pi} \iint\left[\frac{y^{\prime}-y}{r^{3}}\left\{\dot{u}_{0} \cos (y, n)-\dot{v}_{0} \cos (x, n)\right\}\right. \\
& \left.+\frac{z^{\prime}-z}{r^{3}}\left\{\dot{u}_{0} \cos (z, n)-\dot{w}_{0} \cos (x, n)\right\}\right] d \Sigma_{0},
\end{aligned}
$$

where the integration expressed by $\iint \ldots d \Sigma_{0}$ has the same meaning as before. 
Also we found

$$
\iint r\left(u_{0} \frac{\partial^{2} r^{-1}}{\partial x^{2}}+v_{0} \frac{\partial^{2} r^{-1}}{\partial x \partial y}+w_{0} \frac{\partial^{2} r^{-1}}{\partial x \partial z}+\frac{\partial r^{-1}}{\partial x} \frac{\partial q_{0}}{\partial r}\right) d S_{1}=\iint \frac{x^{\prime}-x}{r^{2}} \Delta_{0} d S_{1},
$$

and a like formula holds when the integration is taken over the part of $S_{2}$ that is within $T$. Further we have

$$
\begin{aligned}
& \frac{x^{\prime}-x}{r^{2}} \Delta_{0}-\frac{1}{r} \frac{\partial u_{0}}{\partial r} \\
& =2\left(\frac{y^{\prime}-y}{r^{2}} \varpi_{0}^{\prime \prime \prime}-\frac{z^{\prime}-z}{r^{2}} \varpi_{0}^{\prime \prime}\right)+\left[\frac{x^{\prime}-x}{r^{2}}\left(\frac{\partial v_{0}}{\partial y^{\prime}}+\frac{\partial w_{0}}{\partial z^{\prime}}\right)-\frac{y^{\prime}-y}{r^{2}} \frac{\partial v_{0}}{\partial x^{\prime}}-\frac{z^{\prime}-z}{r^{2}} \frac{\partial w_{0}}{\partial x^{\prime}}\right],
\end{aligned}
$$

and the integral of the terms in square brackets over the part of $S_{1}$ or $S_{2}$ that is within $T$ vanishes. We may therefore write the expression for $u$ in the form

$$
\begin{aligned}
u= & \frac{1}{2 \pi} \iiint\left\{\frac{y^{\prime}-y}{r^{3}}\left(t \dot{\varpi}_{0}^{\prime \prime}+\varpi_{0}^{\prime \prime \prime}\right)-\frac{z^{\prime}-z}{r^{3}}\left(t \dot{\varpi}_{0}^{\prime \prime}+\varpi_{0}^{\prime \prime}\right)\right\} d V \\
& +\frac{1}{2 \pi} \iint\left(\frac{y^{\prime}-y}{r^{2}} \varpi_{0}^{\prime \prime}-\frac{z^{\prime}-z}{r^{2}} \varpi_{0}^{\prime \prime}\right) d S_{1}-\frac{1}{2 \pi} \iint\left(\frac{y^{\prime}-y}{r^{2}} \varpi_{0}^{m}-\frac{z^{\prime}-z}{r^{2}} \varpi_{0}^{\prime \prime}\right) d S_{2} \\
& +\frac{t}{4 \pi} \iint\left[\frac{y^{\prime}-y}{r^{3}}\left\{\dot{u}_{0} \cos (y, n)-\dot{v}_{0} \cos (x, n)\right\}\right. \\
& \left.\quad+\frac{z^{\prime}-z}{r^{3}}\left\{\dot{u}_{0} \cos (z, n)-\dot{w}_{0} \cos (x, n)\right\}\right] d \Sigma_{0} \\
& +\frac{1}{4 \pi} \iint \frac{1}{r^{2}}\left(t \dot{u}_{0}+u_{0}+r \frac{\partial u_{0}}{\partial r}\right) d S_{1} .
\end{aligned}
$$

This equation shows the dependence of the motion in the rotational wave, and between the two waves, upon the initial rotation and velocity of rotation, and upon the tangential components of the initial velocity at the bounuary $\Sigma_{0}$ of $T$. It shows also that, if there is no initial rotation or velocity of rotation, and if the tangential components of the initial velocity at the surface $\Sigma_{0}$ vanish, the dilatational wave alone is propagated, and the displacement in it is expressed in terms of the initial displacement and velocity by means of Poisson's integral formula, as we know it ought to be. In this case the velocity with which the boundary of the disturbed portion of the medium advances is the velocity $a$.

30. Discontinuities at Wave-Fronts.-Expressions for the components of velocity, strain, and stress at any point may be formed in the same 
manner as the expressions for the dilatation and the components of the rotation, by differentiating the expressions such as (64) for the components of displacement. The possibility of non-vanishing values for the velocity, dilatation, rotation, \&c., at the boundary of an advancing wave is bound up with the occurrence of line integrals in the expressions for these quantities, but the magnitudes of these quantities at such wave-fronts cannot be determined by the formulæ already obtained, because, when the point $(x, y, z)$ is on a wave-front at time $t$, one of the spheres $S_{1}, S_{2}$ touches the surface $\Sigma_{0}$, and thus the formulæ (56) and (57) obtained in Article 21 become nugatory. We have to determine the forms of the differential coefficients of $\iint \psi d S_{1}$, say, at an instant when $S_{1}$ touches $\Sigma_{0}$.

We take as the standard case that in which the point $O$ or $(x, y, z)$ is outside $T$, and the sphere of radius at with this point as centre touches $\Sigma_{0}$ at the point $P$, which is the nearest point of $\Sigma_{0}$ to $(x, y, z)$. [Cf. Fig. 6 in Article 32.] Then the sphere of radius $a(t+\delta t)$ with its centre at the same point will cut $\Sigma_{0}$ in a small closed curve lying near to the point $P$. We take temporary axes of $x_{1}, y_{1}, z_{1}$, so that the origin is at the point $P$, and the axis of $z_{1}$ in the positive sense is drawn from $P$ to 0 . The equation of $\Sigma_{0}$ near to $P$ may be written

$$
2 z_{1}=\frac{x_{1}^{2}}{\rho_{1}}+\frac{y_{1}^{2}}{\rho_{2}}
$$

where $\rho_{1}$ and $\rho_{2}$ are the principal radii of curvature of $\Sigma_{0}$ at $P$, and these are estimated as positive when the $z_{1}$-coordinates of the corresponding centres of curvature are positive. In the figure $\rho_{1}$ and $\rho_{2}$ are negative. The equation of $S_{1}$ is $x_{1}^{2}+y_{1}^{2}+\left(z_{1}-r\right)^{2}=r^{2}$; and that of the sphere of radius $a(t+\delta t)$ is given, with sufficient approximation, by changing $r^{2}$ on the right-hand side to $r^{2}+2 a r \delta t$. This sphere cuts $\Sigma_{0}$ in a curve of which the projection on the plane of $\left(x_{1}, y_{1}\right)$ is given, with sufficient approximation, by the equation

$$
x_{1}^{2}+y_{1}^{2}-r\left(\frac{x_{1}^{2}}{\rho_{1}}+\frac{y_{1}^{2}}{\rho_{2}}\right)=2 a r \delta t ;
$$

and the area of the part of the sphere of radius $a(t+\delta t)$ which is included within this curve is, with sufficient approximation, equal to

$$
\left\{\left(1-\frac{r}{\rho_{1}}\right)\left(1-\frac{r}{\rho_{2}}\right)\right\}^{-1} 2 \pi \operatorname{ar} \delta t .
$$

The quantity is real in the standard case, and thus we find the progressive 
differential coefficient of $\iint \psi d S_{1}$ with respect to $t$ at the instant of contact in the form

$$
\frac{\partial}{\partial t} \iint \psi d S_{1}=\frac{2 \pi \operatorname{ar} \psi(P)}{\left\{\left(1-r / \rho_{1}\right)\left(1-r / \rho_{2}\right)\right\}^{\frac{1}{3}}} .
$$

This formula may be extended to any case in which the quantity in the denominator is real: for example, it may be extended to the case where $P$ is the most distant point of $\Sigma_{0}$ from $(x, y, z)$. In that case the sphere of radius $a(t+\delta t)$ would cut $\Sigma_{0}$ if $\delta t$ were negative, and the differential coefficient is then regressive. A similar formula may be obtained in cases where $S_{2}$ touches $\Sigma_{0}$.

Slight changes in the details of the argument are required in determining the effect of contact on the spatial differential coefficients of the surface integrals. Taking, for example, the standard case of contact of $S_{1}$ and $\Sigma_{0}$, the shifting of the sphere $S_{1}$ through a positive $\delta x$ may result in the surfaces cutting one another, and then there is a progressive differential coefficient of $\iint \psi d S_{1}$ with respect to $x$, and it may be proved that

$$
\frac{\partial}{\partial x} \iint \psi d S_{1}=-\frac{2 \pi r \cos (x, n) \psi(P)}{\left\{\left(1-r / \rho_{1}\right)\left(1-r / \rho_{2}\right)\right\}^{\frac{3}{2}}}
$$

and a like formula may be obtained for $\iint \psi d S_{2}$.

By means of the formulæ which have now been obtained, we may form expressions for the components of velocity, strain, and stress, at points on the fronts and rears of the waves of dilatation and rotation, and we may verify that equations of the types of (1) and (2) in Article 3 are satisfied at the front of the wave of dilatation and at the rear of the wave of rotation, and that equations of the types of (3) and (4) are satisfied at the rear of the wave of dilatation and at the front of the wave of rotation.

31. Tendency to the Formation of Surfaces of Discontinuity.-The reality of the denominator in the two expressions (87) and (88) is secured if the point of contact is the nearest point of $\Sigma_{0}$ to $(x, y, z)$ or the most distant point of $\Sigma_{0}$ from $(x, y, z)$; for in these two cases the sphere $\left(S_{1}\right.$ or $\left.S_{2}\right)$ cannot cut $\Sigma_{0}$, but merely touches it. In these cases $r$ is less than the less or greater than the greater of $\rho_{1}$ and $\rho_{2}$, when these are positive. Here $r$ denotes the distance of a point $(x, y, z)$ from a point on the surface $\Sigma_{0}$, and the line joining the points is normal to the surface. If we observe the variations in the formulæ (87) and (88) when the point $(x, y, z)$ moves along a normal to the surface $\Sigma_{0}$, we see that, as $(x, y, z)$ approaches a centre of principal 
curvature of $\Sigma_{0}$, the right-hand members of (87) and (88) tend to become infinite, and that, in passing from one side to the other of the centre of curvature, a discontinuity is encountered. This tendency to discontinuity, leading to infinite velocities and infinite strains, and therefore to rupture of the material, is confined to the immediate neighbourhood of the centrosurface of $\Sigma_{0}$. It is of the same nature as the tendency to infinite pressure at the centre of spherical waves of sound. In the latter case we know that the tendency may be counteracted by a proper adjustment of initial conditions, and it is important to ascertain the conditions which are necessary in the present problem in order that discontinuity of this kind may be avoided.

32. Conditions to be satisfied at the Initial Boundary.-To avoid the tendency to discontinuity which has just been explained, it is sufficient that the quantity which has to be integrated over the small part of $S_{1}$ or $S_{2}$, near its point of contact with $\Sigma_{0}$, should vanish when the centre of $S_{1}$ and $S_{2}$ is one of the centres of principal curvature of $\Sigma_{0}$ at the point of contact. Now we are concerned with the differential coefficients of $u, v, w$ with respect to $x, y, z, t$, and the quantity which has to be integrated over $S_{1}$ or $S_{2}$ may be simplified by omitting the terms that contain $u_{0}, v_{0}, w_{0}, q_{0}$, since these quantities vanish at $\Sigma_{0}$. The following conditions are sufficient, and are also in general necessary, to secure that no discontinuity shall arise :-

(i.) At a point of contact of $\Sigma_{0}$ with a sphere $S_{1}$ having its centre at a centre of principal curvature of $\Sigma_{0}$ the quantity $t \dot{q}_{0}+r \partial q_{0} / \partial r$ vanishes.

(ii.) At a point of contact of $\Sigma_{0}$ with a sphere $S_{2}$ having its centre at a centre of principal curvature of $\Sigma_{0}$ the three quantities of the type

vanish.

$$
\left(t \dot{q}_{0}+r \frac{\partial q_{0}}{\partial r}\right) \frac{\partial r^{-1}}{\partial x}-\frac{1}{r^{2}}\left(t \dot{u}_{0}+r \frac{\partial u_{0}}{\partial r}\right)
$$

These conditions hold, of course, for different values of $t$.

Now, we have, when $r=a t$,

$$
\begin{aligned}
t \dot{q}_{0}+r \frac{\partial q_{0}}{\partial r}=r\left\{\left(\frac{\dot{u}_{0}}{a}+\frac{\partial u_{0}}{\partial r}\right) \cos (r, x)\right. & +\left(\frac{\dot{v}_{0}}{a}+\frac{\partial v_{0}}{\partial r}\right) \cos (r, y) \\
& \left.+\left(\frac{\dot{w}_{0}}{a}+\frac{\partial w_{0}}{\partial r}\right) \cos (r, z)\right\} ;
\end{aligned}
$$

and, when $(x, y, z)$ is a centre of curvature of $\Sigma_{0}$ at $\left(x^{\prime}, y^{\prime}, z^{\prime}\right)$, the direction 
of $r$ is either the same as that of $n$ or opposite to it. To fix ideas, we may take $\Sigma_{0}$ to be a single closed surface which is everywhere convex ; then, at any contact of $S_{1}$ with $\Sigma_{0}$ in which at can be equal to $\rho_{1}$ or $\rho_{2}$, the direction of $r$ is necessarily that of $n$, and we must have

$$
\begin{aligned}
\left(\frac{\dot{u}_{0}}{a}+\frac{\partial u_{0}}{\partial n}\right) \cos (n, x) & +\left(\frac{\dot{v}_{0}}{a}+\frac{\partial v_{0}}{\partial n}\right) \cos (n, y) \\
& +\left(\frac{\dot{w}_{0}}{a}+\frac{\partial w_{0}}{\partial n}\right) \cos (n, z)=0 .
\end{aligned}
$$

On introducing the results (50) we find that this equation becomes

$$
\dot{u}_{0} \cos (x, n)+\dot{v}_{0} \cos (y, n)+\dot{w}_{0} \cos (z, n)=-a \Delta_{0} ;
$$

so that the initial velocity in the direction of the normal at any point of $\Sigma_{0}$ must have the value here given in terms of the initial dilatation at the point.

Again we have, when $r=b t$,

$$
\begin{aligned}
& {\left[\left(t \dot{q}+r \frac{\partial q_{0}}{\partial r}\right) \frac{\partial r^{-1}}{\partial x}-\frac{1}{r^{2}}\left(t \dot{u}_{0}+r \frac{\partial u_{0}}{\partial r}\right)\right]} \\
& =\frac{\left(x^{\prime}-x\right)^{2}}{r^{3}}\left(\frac{\dot{u}_{0}}{b}+\frac{\partial u_{0}}{\partial r}\right)+\frac{\left(x^{\prime}-x\right)\left(y^{\prime}-y\right)}{r^{3}}\left(\frac{\dot{v}_{0}}{b}+\frac{\partial v_{0}}{\partial r}\right) \\
& \quad+\frac{\left(x^{\prime}-x\right)\left(z^{\prime}-z\right)}{r^{3}}\left(\frac{\dot{w}_{0}}{b}+\frac{\partial w_{0}}{\partial r}\right)-\left(\frac{\dot{u}_{0}}{b}+\frac{\partial u_{0}}{\partial r}\right)\left(\frac{\left(x^{\prime}-x\right)^{2}}{r^{3}}+\frac{\left(y^{\prime}-y\right)^{2}}{r^{3}}+\frac{\left(z^{\prime}-z\right)^{2}}{r^{3}}\right) \\
& =\frac{y^{\prime}-y}{r^{2}}\left\{\frac{x^{\prime}-x}{r}\left(\frac{\dot{v_{0}}}{b}+\frac{\partial v_{0}}{\partial r}\right)-\frac{y^{\prime}-y}{r}\left(\frac{\dot{u}_{0}}{b}+\frac{\partial u_{0}}{\partial r}\right)\right\} \\
& \quad+\frac{z^{\prime}-z}{r^{2}}\left\{\frac{x^{\prime}-x}{r}\left(\frac{\dot{w}_{0}}{b}+\frac{\partial w_{0}}{\partial r}\right)-\frac{z^{\prime}-z}{r}\left(\frac{\dot{u}_{0}}{b}+\frac{\partial u_{0}}{\partial r}\right)\right\} ;
\end{aligned}
$$

and, to make all the expressions of this type vanish, when $n$ and $r$ have either the same direction or the opposite direction, we must have

$$
\frac{\dot{u}_{0}}{b}+\frac{\partial u_{0}}{\partial r}: \frac{\dot{v}_{0}}{b}+\frac{\partial v_{0}}{\partial r}: \frac{\dot{w}_{0}}{b}+\frac{\partial w_{0}}{\partial r}=\cos (n, x): \cos (n, y): \cos (n, z) .
$$

On introducing the results (50), and taking the directions of $n$ and $r$ to be the same, we obtain three equations of the type

$$
\dot{v}_{0} \cos (n, z)-\dot{w}_{0} \cos (n, y)=2 b \varpi_{0}^{\prime} ;
$$

so that the tangential components of the initial velocity at points on $\Sigma_{0}$ must be connected with the initial rotation by relations of the type (90). 
The equations (89) and (90) hold when $r$ and $n$ are in the same direction; when they are opposite in direction the signs of the left-hand members must be changed. The geometrical relations involved may be summed up as follows :-Take any point $O$ on the centro-surface of $\Sigma_{0}$, and draw from it the normal to $\Sigma_{0}$ at that point $(P)$ of $\Sigma_{0}$ for which $O$ is a centre of principal curvature: when this normal, in this sense, passes at $P$ from the region $T$ within $\Sigma_{0}$ to the region outside $\Sigma_{0}, r$ and $n$ have the same sense; otherwise they have the opposite sense.

It follows that, if $\Sigma_{0}$ is synclastic near a point $P$, one or other of the two relations

$$
\pm\left\{\dot{u}_{0} \cos (x, n)+\dot{v}_{0} \cos (y, n)+\dot{w}_{0} \cos (z, n)\right\}=-a \Delta_{0}
$$

holds at $P$, and also one or other of the two sets of relations of the type

$$
\pm\left\{\dot{v}_{0} \cos (z, n)-\dot{v}_{0} \cos (y, n)\right\}=2 b \varpi_{0}^{\prime},
$$

and the ambiguous sign is the same for all these relations, viz., it is + when the normal drawn from the centre of curvature passes from within $T$ to outside $T$. But when the surface $\Sigma_{0}$ is anticlastic near $P$ the relations of type (91) and those of type (92) must in general hold with both signs. In the latter case (anticlastic) the initial velocity must vanish at $P$ and likewise the initial dilatation and rotation must vanish at $P$. In the case where $\Sigma_{0}$ is synclastic at $P$ the initial velocity, dilatation, and rotation need not vanish at $P$; but the normal component of the velocity must be "compensated" by the initial dilatation, and the tangential components of the velocity must be "compensated" by the initial rotation.

33. Application of these conditions to the case of Spherical Waves of Sound.-It seems worth while to illustrate the necessity of the conditions of type (91) and (92) by reference to the problem of spherical waves of sound. In the simplest case which can serve as an illustration, the motion is confined initially to the volume within a sphere $\Sigma_{0}$, and the motion is symmetrical about the centre of the sphere. Let $C$ be the centre of $\Sigma_{0}$ and $c$ its radius, and let $\phi$ be the velocity potential of the motion and $a$ the velocity of sound. Then $\phi$ is a function of $r$, the distance of any point from $C$, and of $t$; and we must have

$$
\phi=r^{-1}\{f(a t-r)+F(a t+r)\},
$$

where $f$ and $F$ are functions which, for positive arguments, are connected by the equation

$$
f(\xi)+F(\xi)=0 ;
$$

for this is the condition that the centre $C$ may not act as a source. 
In the initial state the velocity, $\partial \phi / \partial r$, and the condensation, $-a^{-2} \partial \phi / \partial t$, are given functions of $r$ in the region $r \leqslant c$, and vanish in the region $r>c$. Let $\phi_{0}$ be the initial value of $\phi$ and $\dot{\phi}_{0}$ that of $\partial \phi / \partial t$. From the nature of the problem $\partial \phi_{0} / \partial r$ must vanish at the centre $C$, and, since $\phi_{0}$ is indeterminate to the extent of an additive arbitrary constant when $\partial \phi_{0} / \partial r$ is given, we may take $\phi_{0}$ to vanish at $r=c$. To simplify the problem as much as possible we shall suppose that in the region $r \leqslant c$ the functions $\partial \phi_{0} / \partial r$ and $\dot{\phi}_{0}$ are analytic and without singularities. We may take $\phi_{0}$ to be determined for all positive values of $r$ as a function $\psi(r)$ which has the following properties :-

(i.) $\psi(r)$ is analytic and without singularities in the region $r \leqslant c$,

(ii.) $\psi(r)=0$ in the region $r>c$,

(iii.) $\psi^{\prime}(r)=0$ at $r=0$,

(iv.) $\psi(r)=0$ at $r=c$.

In like manner we may take $\phi_{0}$ to be determined for all positive ralues of $r$ as a function $\Psi_{1}(r)$ which has the following properties :-

(i.) $\Psi_{1}(r)$ is analytic and without singularities in the region $r \leqslant c$.

(ii.) $\Psi_{1}(r)=0$ in the region of $r>c$.

It is convenient to introduce a new function $\Psi(r)$ by the definitions

$$
\begin{array}{ll}
\text { (i.) } \Psi(r)=-\int_{r}^{c} \xi \Psi_{1}(\xi) d \xi & \text { in the region } r \leqslant c, \\
\text { (ii.) } \Psi(r)=0 & \text { in the region } r>c .
\end{array}
$$

Then $\dot{\phi}_{0}=r^{-1} \Psi^{\prime}(r)$, and $\Psi^{\prime}(r)$ vanishes at $r=0$. At $r=c$ the derivatives $\psi^{\prime}(c)$ and $\Psi^{\prime}(c)$ must be understood to be regressive, and at $r=0$ the derivatives $\psi^{\prime}(0)$ and $\Psi^{\prime}(0)$ must be understood to be progressive.

The functions $f$ and $F$ are now to be determined for all values of their arguments which can come into consideration by the equation (94) and the equations

$$
F(r)+f(-r)=r \psi(r), \quad F^{\prime}(r)+f^{\prime}(-r)=a^{-1} \Psi^{\prime}(r),
$$

the first of which gives $F^{\prime \prime}(r)-f^{\prime}(-r)=\psi(r)+r \psi^{\prime}(r)$. For all positive values of $\xi$ the functions $F^{\prime \prime}(\xi)$ and $f^{\prime}(\xi)$ are given by the equations

$$
\begin{aligned}
F^{\prime}(\xi) & =\frac{1}{2}\left[\psi(\xi)+\xi \psi^{\prime}(\xi)+a^{-1} \Psi^{\prime}(\xi)\right], \\
f^{\prime}(\xi) & =-F^{\prime}(\xi),
\end{aligned}
$$

of which the second is derived from (94).

Consider the value of the condensation at a point $O$ near to $C$, and at Sse. 2. voL. 1. No. 841 . 
an instant after the inwards travelling wave has reached the point $O$ but before the motion at the point $O$ has ceased. At such an instant we have $c+r>a t>c-r$, and we may put, therefore, $a t=c+r-2 r \cdot$, where $\mathcal{Q}$ is a positive number less than unity. At any point $O$ within $\Sigma_{0}$, other than $C$, the interval in question is finite, and $q$ runs through all values from 1 to 0 , although the interval diminishes indefinitely as $O$ moves towards $C$. The condensation at $O$ is $-(a r)^{-1}\left\{F^{\prime \prime}(a t+r)+f^{\prime}(a t-r)\right\}$. Now at $+r=c+2 r(1-q)$, which is $>c$, and thus $F^{\prime \prime}(a t+r)$ vanishes by (95) and the definitions of $\psi$ and $\Psi$. Also $a t-r=c-2 r$, , and thus $(95)$ and $(96)$ give

$$
f^{\prime}(a t-r)=-!\left[\psi \left[(c-2 \cdot 9)+\left(c-2 \gamma \cdot 9 \gamma^{\prime}(c-2 r \cdot 9)+a^{-1} \Psi^{\prime}(c-2 r \cdot q)\right] .\right.\right.
$$

Since $y_{r}^{\prime}(c)=0$, this expression tends to the limit $-l_{2}^{1}\left({ }^{\prime}\left(\psi^{\prime}(c)+a^{-1} \Psi^{\prime}(c){ }_{i}^{\prime}\right.\right.$ as $r$ teuds to the limit zero. Hence it follows that the condensation near $C$ tends to become infinite * in the order $?_{2}^{\prime}(a r)^{-1}\left\{c c^{\prime} r^{\prime}(c)+a^{-1} \Psi^{\prime}(c)\right\}$ unless

$$
c \psi^{\prime}(c)+a^{-1} \Psi^{\prime}(c)=0 .
$$

This equation is equivalent to the equation

$$
a\left(\frac{\hat{c} \phi_{0}}{\hat{r})_{i}}\right)_{r=c}+\left(\dot{\phi}_{0}\right)_{r=c}=0
$$

which is the analogue of (91) and (92) for the problem in hand. In terms of initial relocity $u_{0}$ and condensation $s_{0}$ it is the same as $u_{0}=a s_{0}$ at $r=c$. I have rerified that the same result would be arrived at by evaluating $\phi$ in terms of $\phi_{0}$ and $\dot{\phi}_{0}$ by means of Poisson's integral formula.* This method shows also that the condition (99) is sufficient to secure that neither the pressure nor the velocity shall tend to become infinite in the neighbourhood of $C$.

The problem of spherical waves of sound symmetrical about the centre of the sphere has been treated by many writers. including Poisson, $\downarrow$ Stokes $\$$ and Lord Rayleigh, but the necessity of the condition here investigated does not appear to have heen noticed.

"At the point $C$ the condensation is of courne fiuite, the infinite linit not being "attained." This peculiarity is of constaut vccurrence in the the theory of non-analytical functions. See, for example. En:y. Sirt. (Swpplemem), Vol. xxvir.. Article "Functions of Real Variables." It may 1, hold that infinite limit: are never attained.

+ Cf. my paper, supia, p. 4 , where, however. the interpretation of the results for points near the entre of the sphere is imperfect.

; Treitc le Micrnique, 2nd edition (Paris, 183:3), t. Ir., pp. 699-708.

\$Phil. May. (Ser. 3), Vul. xxxrv. (1849), or Math. and Phys. Papers, Vol. Ir., p. 83.

it 7heory of Sound, Vol. II., pp. 109-111. 
34. Simplification of the Results by the Conditions at the Initial Boundary.-The relations between initial velocities and initial dilatations and rotations at the boundary $\Sigma_{0}$ of $T$, which have now been shown to be necessary in general in order to avoid dangerous discontinuities and rupture of the material, may be used to simplify the expressions obtained in Articles 27 to 29. For example, when $\Sigma_{0}$ is a single closed surface which is everywhere convex, we have for the dilatation

$$
\Delta=\frac{1}{4 \pi} \iint \frac{1}{r^{2}}\left(t \dot{\Delta}_{0}+\Delta_{0}+r \frac{\partial \Delta_{0}}{\partial r}\right) d S_{1}+\frac{1}{4 \pi} \int \frac{1-\cos (r, n)}{r \sin \chi} \Delta_{0} d s_{1},
$$

and for the rotation we have such equations as

$$
2 \varpi^{\prime}=\frac{1}{2 \pi} \iint \frac{1}{r^{2}}\left(t \dot{\varpi}_{0}^{\prime}+\varpi_{0}^{\prime}+r \frac{\partial \varpi_{0}^{\prime}}{\partial r}\right) d S_{2},+\frac{1}{2 \pi} \int \frac{1-\cos (r, n)}{r \sin \chi} \varpi_{0}^{\prime} d s_{2} .
$$

For the displacement we have alternative formula of the types

$$
\begin{aligned}
u= & \frac{1}{4 \pi} \iint \frac{x^{\prime}-x}{r^{3}}\left(t \dot{\Delta}_{0}+\Delta_{0}\right) d V+\frac{1}{4 \pi} \iint \frac{x^{\prime}-x}{r^{2}} \Delta_{0} d S_{1}-\frac{1}{4 \pi} \iint \frac{x^{\prime}-x}{r^{2}} \Delta_{0} d S_{2} \\
& +\frac{a t}{4 \pi} \iint \frac{x^{\prime}-x}{r^{3}} \Delta_{0} d \Sigma_{0}+\frac{1}{4 \pi} \iint \frac{1}{r^{2}}\left(t \dot{u}_{0}+u_{0}+r \frac{\partial u_{0}}{\partial r}\right) d S_{2}
\end{aligned}
$$

and

$$
\begin{aligned}
u= & \frac{1}{2 \pi} \iiint\left\{\frac{y^{\prime}-y}{r^{3}}\left(\dot{t}_{0}^{\prime \prime \prime}+\varpi_{0}^{\prime \prime \prime}\right)-\frac{z^{\prime}-z}{r^{3}}\left(t \dot{\varpi}_{0}^{\prime \prime}+\varpi_{0}^{\prime \prime}\right)\right\} d V \\
& +\frac{1}{2 \pi} \iint\left(\frac{y^{\prime}-y}{r^{2}} \varpi_{0}^{\prime \prime \prime}-\frac{z^{\prime}-z}{r^{2}} \varpi_{0}^{\prime \prime}\right) d S_{1}-\frac{1}{2 \pi} \iint\left(\frac{y^{\prime}-y}{r^{2}} \varpi_{0}^{\prime \prime \prime}-\frac{z^{\prime}-z}{r^{2}} \varpi_{n !}^{\prime \prime}\right) d S_{2} \\
& +\frac{b t}{2 \pi} \iint\left(\frac{y^{\prime}-y}{r^{3}} \varpi_{0}^{\prime \prime \prime}-\frac{z^{\prime}-z}{r^{3}} \varpi_{1 \prime \prime}^{\prime \prime}\right) d \Sigma_{0}+\frac{1}{4 \pi} \iint \frac{1}{r^{2}}\left(t \dot{u}_{0}+u_{0}+r \cdot \frac{\partial u_{0}}{\partial r}\right) d S_{1} .
\end{aligned}
$$

Like results with suitable modifications hold also when $\Sigma_{0}$ is not of the above simple character; in all cases the normal aud tangential components of the initial velocity at points on $\Sigma_{0}$ can be replaced by quantities of the form $\pm a \Delta_{0}, \pm 2 b \varpi_{0}^{\prime}, \ldots$ It appears that, in all cases, the dilatation can be expressed in terms of the initial dilatation and velocity of dilatation, and the rotation can be expressed in terms of the initial rotation and velocity of rotation; but the expressions are not identical with those given by Poisson's integral formula unless the dilatation, in the one case, and the rotation, in the other, vanish at $\Sigma_{0}$. It has been shown further that the 
displacement can be resolved into two constituent displacements in two alternative ways. In one way, one of the constituents depends entirely on the initial dilatation and velocity of dilatation, and the other constituent is expressed, in terms of initial displacement and velocity, by the formulæ that would express the displacement if a rotational wave alone were propagated. In the other mode of resolution, one of the constituents depends entirely on the initial rotation and relocity of rotation, and the other is expressed, in terms of initial displacement and velocity, by the formulæ that would express the displacement if a dilatational wave alone were propagated. At places which are so distant from $T$ that the rear of the wave of dilatation has passed before the front of the wave of rotation arrives, the motion in the wave of dilatation depends on the initial dilatation and velocity of dilatation only, and the motion in the wave of rotation depends on the initial rotation and velocity of rotation only.

The initial displacement and velocity may involve dilatation without rotation or velocity of rotation throughout the region $T$. In this case the conditions that no discontinuity leading to rupture of the material may arise are that the displacement vanishes at the boundary $\Sigma_{0}$ of $T$, and that, at the same surface, the initial velocity is directed along the normal to $\Sigma_{0}$, and is equal to the value of $a \Delta_{0}$ at each point of $\Sigma_{0}$. In this case a wave of dilatation only will be propagated, and the displacement in it will be determined by Poisson's integral formula. Again the initial displacement and velocity may involve rotation without dilatation or velocity of dilatation throughout the region $T$, and the components of the displacement and the normal component of the initial velocity may vanish at all points of $\Sigma_{0}$. In this case, if the tangential components of velocity are adjusted in accordance with the conditions (92), a rotational wave only will be propagated, and the displacement in it will be determined by Poisson's integral formula. A more general type of initial conditions can be arrived at by superposing the displacements and velocities in two initial states of the two kinds just described. But this type is not the most general possible type of initial conditions. It is true that any initial displacement and velocity may be resolved into two systems of displacement and velocity, of which one involves no rotation and the other no dilatation. This resolution would be effected by the intervention of scalar potentials and vector potentials. But, in general, the resolution of the displacement cannot be effected so that the displacements of both systems vanish at $\Sigma_{0}$; and the resolution of the velocity cannot be effected so that the velocity of one system shall be normal to $\Sigma_{0}$, and have a given value at each point on $\Sigma_{0}$, while the velocity of the other system is tangential to $\Sigma_{0}$, and has a given magnitude and direction at each point on $\Sigma_{0}$. 
35. Dilatation and Rotation at the Fronts and Rears of Wavcs.We take the case in which $\Sigma_{0}$ is a single closed surface which is everywhere convex, and take any point $O$ outside it which is not a centre of principal curvature at any point of it. Let $P$ and $Q$ be the points of $\Sigma_{0}$ which are respectively nearest to $O$ and furthest from $O$, as shown in Fig. 6 of Article 32, and let $\rho_{1}^{\prime}$, $\rho_{2}^{\prime}$ be the numerical values of the principal radii of curvature at $P$, and $\rho_{1}^{\prime \prime}, \rho_{2}^{\prime \prime}$ those of the principal radii of curvature at $Q$. A sphere with centre at $O$ and radius less than $O P$ or greater than $O Q$ does not cut $\Sigma_{0}$. When the sphere is of radius $O P$, the denominator in the formulæ (87) and (88) must be replaced by $\left\{\left(1+r / \rho_{1}^{\prime}\right)\left(1+r / \rho_{2}^{\prime}\right)\right\}^{3}$; when its radius is $O Q$, the denominator in these formulæ becomes $\left\{\left(r / \rho_{1}^{\prime \prime}-1\right)\left(r / \rho_{2}^{\prime \prime}-1\right)\right\}^{\frac{1}{3}}$, and this is necessarily real.

When $a t=O P$, the sphere $S_{1}$ touches $\Sigma_{0}$ at $P$, and the dilatation at $O$ when the wave reaches it is obtained from the formulæ of type (64) by the rules of differentiation such as (88). Now $n$ and $r$ lave opposite signs, and the expression $\dot{q}_{0} / a+\partial q_{0} / \partial r$ may be replaced by

$$
-a^{-1}\left\{\dot{u}_{0} \cos (x, n)+\dot{v}_{0} \cos (y, n)+\dot{v}_{0} \cos (z, n)\right\}+\Delta_{0}
$$

which is $2 \Delta_{0}(P)$, and it follows that the dilatation at $O$ when the front of the wave of dilatation reaches $O$ is given by the equation

$$
\Delta=\frac{i}{i}\left(1+\frac{r}{\rho_{1}^{\prime}}\right)\left(1+\frac{r}{\rho_{2}^{\prime}}\right) i^{i-\frac{1}{-}} \Delta_{0}(P)
$$

in which $r=a t$.

When $a t=O Q$, the sphere $S_{1}$ touches $\Sigma_{0}$ at $Q$, and the dilatation at $O$ just before the rear of the wave of dilatation passes over $O$ is found, by the above process and by paying attention to the signs, to be zero.

It appears in like manner that, when $b t=O P$, so that the front of the wave of rotation has just reached $O$, the values of the components of rotation at $O$ are given by such formulæ as

$$
\varpi^{\prime}=\left\{\left(1+\frac{r}{\rho_{1}^{\prime}}\right)\left(1+\frac{r}{\rho_{2}^{\prime}}\right)\right\}^{-\frac{1}{2}} \varpi_{0}^{\prime}(P) ;
$$

but, when the rear of the wave of rotation is just about to pass over $O$, the rotation at $O$ is reduced to zero.

It may be observed that, if $O$ is so near to $\Sigma_{0}$ that the waves are practically plane, the dilatation at the front of the wave of dilatation, and the rotation at the front of the wave of rotation, are practically the same as the initial dilatation and rotation at the surface $\Sigma_{0}$. 
36. Summary.-The theory of this paper rests upon certain integrals of the equations of motion of an elastic solid medium under the action of body forces. These integrals have in effect been known for a long time, and the proof of their correctness has here been recapitulated briefly. The results which can be obtained by regarding the forces as operative at a single point have been considered in detail. Besides the direct proof, various verifications have been adduced, and the exact nature of the motion specified by the integrals in particular cases has been discussed with graphic illustrations. From the results in the case of a single force have been deduced those that belong to various kinds of double and multiple forces, and it is found that various well-known types of wavemotion can be regarded as due to singularities of these kinds. Among the types of wave-motion in an elastic solid which have been expressed in terms of singularities are the simplest type of dilatational waves, the simplest type of rotational waves and the type of rotational waves in which the displacement is expressed by the same formulæ as were given by Hertz to represent the electric field around his oscillator. From a knowledge of the effects produced by force at a point and of certain types of double forces it was possible to obtain a theorem which is a generalization of Kirchhoff's version of Huygens' principle. In Kirchhoff's theorem, the function which represents the disturbance in wave-motion, determined by a single partial differential equation, is expressed in terms of sources and doublets distributed over a surface. The sources depend on the normal gradient of the function, and the doublets on the value of the function itself, at points on the surface, and at instants determined by the condition that the effects travel from the surface to any point with the appropriate waverelocity. In the present case the two sorts of singularities are points at which forces act and points at which there are double forces of a certain general type. The forces are the actual tractions across the elements of the surface, and the donble forces depend upon the displacements at points of the surface, but the instants at which the forces and double forces must be calculated are not determined by a single wave-velocity. It is as if some effects travelled through the medium with velocities intermediate between that of rotational waves and that of dilatational waves, while other particular effects travel with those definite velocities. Various results enforce this conclusion.

The problem of the propagation of waves due to given initial conditions is taken up, and at the outset it is assumed that the initial disturbance is confined to a region of space occupying a finite volume. Within this region it is assumed that the components of the initial displacement and velocity are expressed by functions which are analytic 
and withont singularities. The condition of continuity of the displacement at the boundary of this region is supposed to be satisfied by the initial displacement, but no other restrictious are imposed upon the initial state. The potentials of the disturbance due to initial velocity are obtained from the results found for force at a point, and the displacement due to initial velocity is deduced. The complete expression for the displacement is then found by means of Stokes's rule. The process involves the determination of rules for the differentiation of integrals taken over portions of moving spherical surfaces which are bounded by the intersections of the spheres with a fixed surface. It is pointed out that the application of these rules of differentiation to Poisson's integral formula brings to light a difference between two forms of it which are sometimes treated as equivalent. The expressions for the displacement which are obtained by the method above described are equivalent to those which were found by Stokes, and previously by Poisson and Ostrograulsky; but the method is different, inasmuch as it takes account at every step of the existence of a boundary to the portion of the medium which is disturbed initially. The importance of this is seen at the next stage of the investigation, when the dilatation and rotation are calculated from the displacement ; for the values of these quantities, when the initial velocity does not vanish at the boundary of the initially disturbed portion of the medium, are not the same as those assumed as a basis of calculation by Stokes. His doctrine, however, of the irrotational and equivoluminal character of the two waves is confirmed. Correct values for the dilatation and rotation are obtained, and some new formule are found for the displacement. By the aid of these formulie the parts played in the motion by the initial dilatation and rotation can be distinguished. The corrections to be made in Stokes's values for the dilatation and rotation depend respectively upon the normal and tangential components of the initial velocity at the boundary of the initially disturbed portion of the medium.

An important question arises as to whether the motion determined by initial conditions, when no restrictions except that of continuity of displacement are imposed at the boundary of the initially disturbed portion of the medium, will or will not result in accumulation of strain at some point, leading to rupture of the material. The question is analogous to one which occurs in the theory of sound. Spherical sound waves may involve a tendency to infinite pressure in the neighbourhood of the centre of the sphere. It is shown that in the present case arbitrary initial conditions will result in such accumulations of strain, and these will occur in the neighbourhood of the points which are centres of principal curvature of the bounding surface of that portion of the medium to which the initial 
disturbance is confined. To avoid such accumulations of strain, conditions must be imposed on the initial state. These conditions take the form of relations between initial velocity and strain at the boundary of the initially disturbed portion of the medium. The normal component of the initial velocity is equal to the product of the velocity of dilatational waves and the initial dilatation. The tangential components are similarly related to the velocity of rotational waves and the initial rotation. These conditions are illustrated by a problem concerning spherical waves of sound. It is shown that, if the initial disturbance in a gaseous medium is confined to the volume within a spherical surface and is symmetrical about the centre of the sphere, the tendency to infinite pressure at the centre is obviated if the initial velocity and condensation at the surface of the sphere are connected by the same relation as holds in the case of plane waves of sound. In the problem of the elastic solid medium, when the like conditions are taken into account it appears that the corrections which must be made in Stokes's formulæ for the dilatation and rotation can be expressed in terms of the dilatation and rotation at the initial boundary. When this boundary is a single closed surface which is everywhere convex, it appears further that the dilatation always vanishes at the rear (though not at the front) of the wave of dilatation, and the rotation always vanishes at the rear of the wave of rotation. 